\title{
Blind image quality assessment based on aesthetic and statistical quality-aware features
}

\author{
Mohsen Jenadeleh,, ${ }^{a, b, *}$ Mohammad Masood Masaeli, ${ }^{a}$ and Mohsen Ebrahimi Moghaddam ${ }^{a}$ \\ ${ }^{a}$ Shahid Beheshti University, Faculty of Computer Science and Engineering, G. C. Tehran, Iran \\ bUniversity of Konstanz, Department of Computer and Information Science, Konstanz, Germany
}

\begin{abstract}
The main goal of image quality assessment (IQA) methods is the emulation of human perceptual image quality judgments. Therefore, the correlation between objective scores of these methods with human perceptual scores is considered as their performance metric. Human judgment of the image quality implicitly includes many factors when assessing perceptual image qualities such as aesthetics, semantics, context, and various types of visual distortions. The main idea of this paper is to use a host of features that are commonly employed in image aesthetics assessment in order to improve blind image quality assessment (BIQA) methods accuracy. We propose an approach that enriches the features of BIQA methods by integrating a host of aesthetics image features with the features of natural image statistics derived from multiple domains. The proposed features have been used for augmenting five different state-of-the-art BIQA methods, which use statistical natural scene statistics features. Experiments were performed on seven benchmark image quality databases. The experimental results showed significant improvement of the accuracy of the methods. (2017 SPIE and IS\&T [DOI: 10 .1117/1.JEI.26.4.043018]
\end{abstract}

Keywords: blind image quality assessment; aesthetic assessment features; quality-aware features; feature enrichment; performance improvement.

Paper 161062 received Dec. 22, 2016; accepted for publication Jul. 20, 2017; published online Aug. 19, 2017.

\section{Introduction}

Image quality assessment (IQA) is considered in many image processing applications such as image enhancement, ${ }^{1}$ contrast preserving decolorization, ${ }^{2}$ image comparison, ${ }^{3}$ and image compression. ${ }^{4-6}$ Traditionally, image quality has been assessed subjectively by human observers. However, subjective evaluation of image quality by humans is reliable but this process is time consuming, expensive, not scalable, and cannot be used in real-time image processing applications. Therefore, objective IQA methods were designed to estimate the subjective image quality scores. To evaluate the performance of an IQA method, the correlation of its objective scores with subjective scores on benchmark IQA databases is often considered. One commonly used summary statistic of subjective scores is the mean opinion score (MOS) obtained from a group of expert judges. Another commonly used summary statistic of the subjective scores is the subtraction of the MOS for a reference image and the MOS for a distorted image, which is called the differential mean opinion score (DMOS).

The no-reference or blind image quality assessment (BIQA) methods aim to assess the quality of an image with no prior information about the reference pristine version. BRISQUE, ${ }^{7}$ NIQE,${ }^{8}$ DIIVINE, ${ }^{9}$ BLIINDS-II, ${ }^{10} \mathrm{M} 3,{ }^{11}$ and BIQWS $^{12}$ are examples of BIQA. The blind methods are close to human traits in image quality assessment and they are more applicable in most areas because most images on the Internet do not have a pristine reference version. The main goal of this paper is to contribute to this category of the image quality assessment methods. Galileo and John Locke

${ }^{*}$ Address all correspondence to: Mohsen Jenadeleh, E-mail: mohsen. jenadeleh@uni-konstanz.de divide the quality into two categories: primary and secondary. According to their idea, primary qualities are genuine properties of things that mathematics can describe, such as shape, motion, and quantity, whereas secondary qualities exist only in human consciousness such as taste, odor, and color. $^{13}$

Humans consider many factors when assessing perceptual image quality. Subjective IQA assessment is based on asking users to report their perceptual quality satisfaction with respect to an image content in specific settings such as viewing distance, light condition, screen size, etc. Human judgments of image quality may also implicitly consider image content and aesthetics of the images. As evidence, Fig. 1 shows two images. These images are from the TID2013 IQA database ${ }^{14}$ with the same distortion type (contrast change) and same severity (both have level 4 in severity), but the subjective quality scores show that the subjects think the left one [Fig. 1(a)] has a higher quality than the other one [Fig. 1(b)].

The effect of image content in perceptual image quality evaluation is well-known. Siahaan et al. ${ }^{15}$ integrated image semantic information to image quality. Their study shows that image semantics influences the perceptual image quality. Image semantics recognition may become more difficult when the image has visual distortions. ${ }^{16}$ Redi $^{17}$ showed that aesthetic appeal is critical in perceptual image quality assessment. However, many BIQA methods use natural scene statistics (NSS) features and emulate subjective quality based on visible impairments of the image distortions and neglect the influence of image content on image quality assessment that limits their performance. They usually

1017-9909/2017/\$25.00 @ 2017 SPIE and IS\&T 


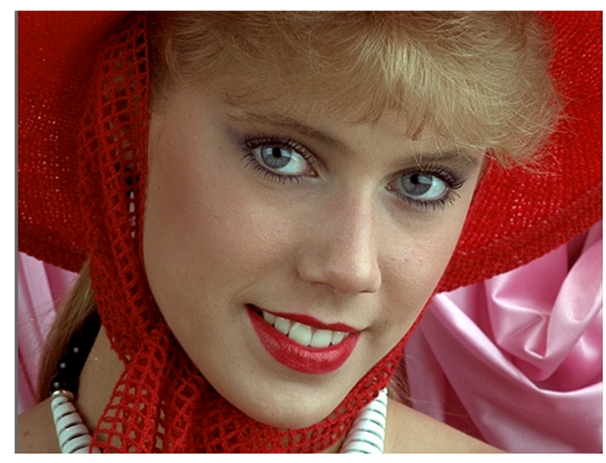

(a)

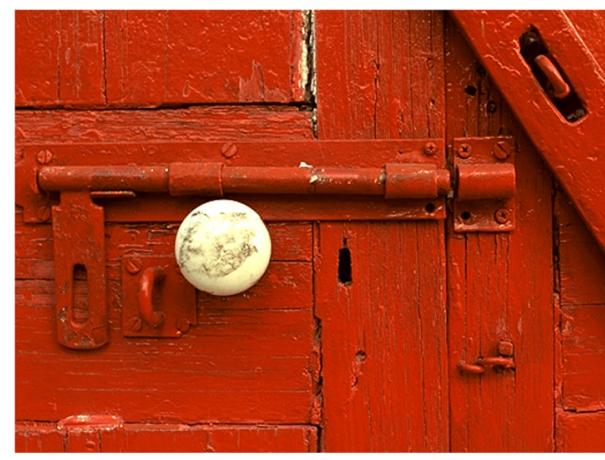

(b)

Fig. 1 Two pictures from TID2013 database ${ }^{14}$ affected by the same distortion (contrast change) with the same severity but the subjective scores differ widely, (a) $80 \%$ and (b) $53 \%$ of scale from $0 \%$ to $100 \%$. This seems to be due to the difference in aesthetics appeal.

measure the quality impairments due to noise distortion, blur, lack of sharpness, dynamic range, contrast, and exposure accuracy.

To overcome this limitation, we proposed a set of image aesthetic features that can enrich the existing NSS features in order to emulate human perceptual quality evaluation more accurately.

To evaluate our approach, the proposed features have been integrated with five different state-of-the-art BIQA methods, which use statistical features and experiments have been performed on seven benchmark image quality databases. In other words, the performance of the original BIQA methods on each IQA database is reported, then the features of the methods are enriched by the proposed aesthetic features with the same training and test subset, then the performance of the enriched methods is evaluated. The experimental results show significant improvement of the accuracy of the methods.

Our contributions can be summarized as follows.

1. We studied extensive aesthetic assessment features from the fields of photoquality assessment and evolutionary art (EA) and modified some of them to correlate better with image quality. From these features, we selected 11 features that are not redundant and correlate well with image quality assessment. These features are described in Sec. 3.

2. Some new experiments have been carried out to support the argument that the observed performance improvements in the enriched BIQA methods are due to the aesthetic features (Sec. 4.4). For this aim, some experiments based on multiobjective feature selection and principal component analysis (PCA) are conducted. Also, we present analysis and visualizations of the psychometric studies for the image quality databases used for our experiments to study the consistency of subjective judgments among human observers.

3. We provide an approach for using aesthetic assessment features in automatic BIQA to improve the performance of the existing blind image quality methods.

4. Extensive experiments have been carried out to provide a statistical verification for performance improvements of enriched BIQA methods using aesthetics assessment features on seven benchmark image quality databases.

The rest of this paper has been organized as follows: in Sec. 2, the related works are presented, proposed aesthetic features are described in Sec. 3, then in Sec. 4 the experiments are conducted to show the performance improvement of the enriched BIQA methods by the proposed aesthetic features. Finally in Sec. 5, the paper is concluded.

\section{Related Work}

In this section, we classify and review the existing objective no-reference or BIQA methods. Saha and $\mathrm{Wu}^{18}$ classified BIQA methods into two main categories: distortion specific and general purpose (or universal) as shown in Fig. 2. The distortion specific methods are based on determining types and severities of the distortion attacks. Some of these methods are developed to assess image quality when a specific distortion such as blur, chromatic aberration, blocking effect, or noise degrades image quality. For example, Kerouh and Serir ${ }^{19}$ proposed an edge analysis-based method in the wavelet transform domain for objective measure of blurred image quality.

In another work, an orthogonal moment-based method ${ }^{20}$ was proposed for no-reference image blur assessment. In this method, the gradient of the input image is computed then output of the gradient is divided into blocks. After that, Tchebichef moments of the blocks are computed and in the final step image score is predicted using variance-normalized moment energy. In another blur distortion specific method, motion blur analysis is employed. ${ }^{21}$ In this method, image sharpness is assessed by the variance and the contrast over the spectral domain.

A no-reference color image quality metric is proposed by Lamb and Khambete ${ }^{22}$ to assess chromatic aberration distortion in color images based on the correlation between the $G$ plane with the $R$ and $B$ planes. Golestaneh and Chandler ${ }^{23}$ also presented a method to assess the quality of JPEG images blindly. They used a quality relevant map of the number of zero-valued discrete cosine transform (DCT) coefficients of image blocks to estimate the effect of JPEG compression. Another method for blind JPEG images quality assessment has been proposed by Gastaldo et al., ${ }^{24}$ which used PCA to achieve a feature vector of input image characterizing the 


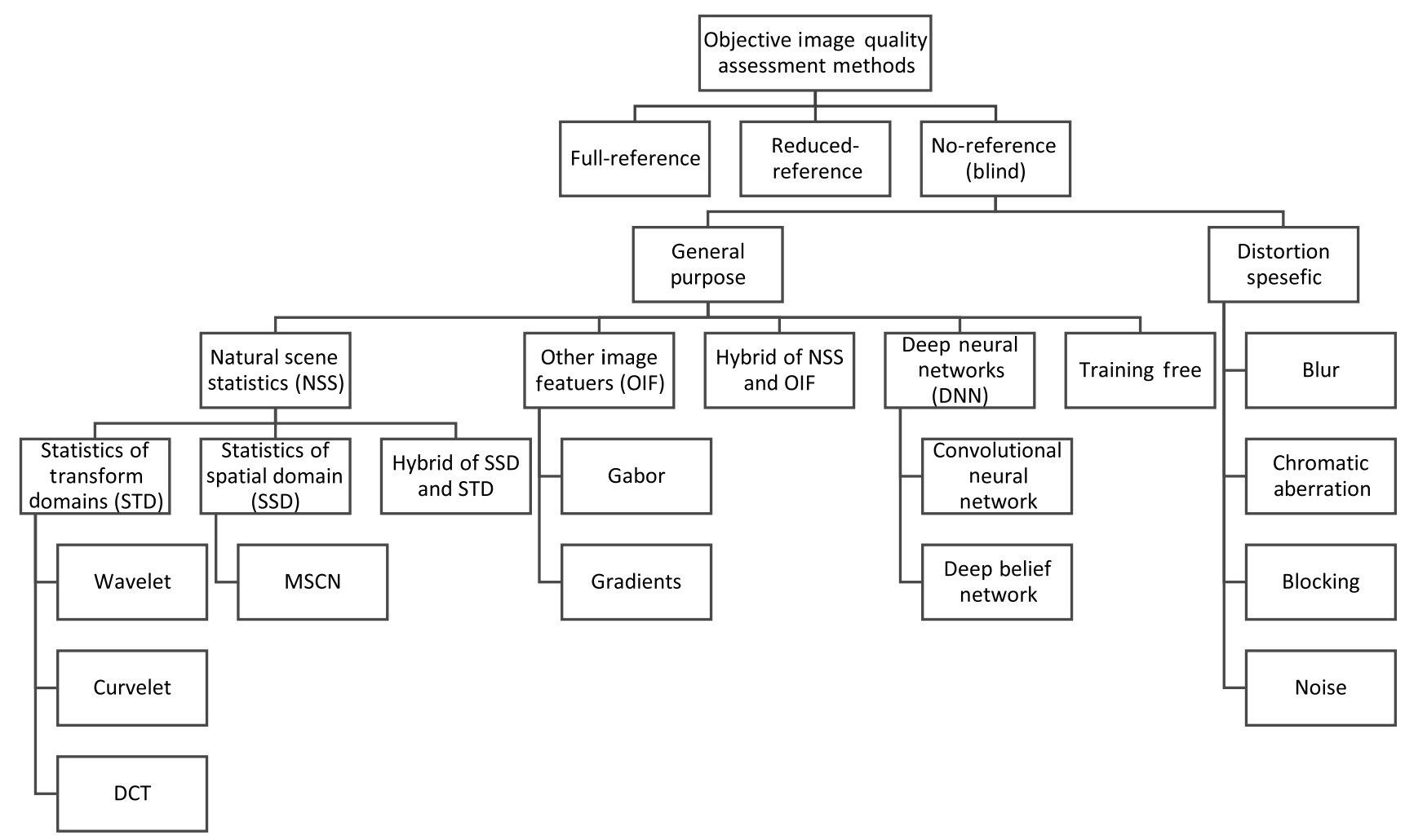

Fig. 2 Classification of objective image quality assessment methods. In this paper, we focus on no-reference (or blind) methods.

information in the image, then a circular back-propagation neural network is used for predicting the test image quality score. Some distortion specific methods have been proposed for assessing JPEG2000 image quality blindly. Sheikh et al. ${ }^{25}$ utilized the statistics of wavelet transform to form a set of quality aware features and then trained a neural network with human opinion scores to predict the test images quality scores.

A structural content-weighted pooling approach for blind JPEG2000 image quality prediction has been proposed by Zhang and $\mathrm{Le}^{26}$, which does not need to be trained by subjective scores. Some methods have been also presented to assess quality of an image in the presence of multiple distortions such as the method proposed by Shen et al. ${ }^{27}$ This approach is an effort to make a hybrid of curvelet, wavelet, and cosine transforms to predict noisy, blurry, JPEG2000, and JPEG image quality score. SISBLIM ${ }^{28}$ is another hybrid method that computes quality for each distortion separately then combines joint effects of each distortion source.

All of the distortion specific BIQA methods are applicable only when the image distortion is known. To overcome this limitation, the general purpose or universal BIQA methods are proposed. The general purpose methods are further classified into five subcategories based on the way of forming their quality-aware feature vectors and predicting the image quality score: NSS, other image features (OIF), hybrid of NSS and OIF, deep neural networks (DNNs), and training free. The methods in the first group use NSS-based qualityaware features and then utilize a neural network to model the complex nonlinear relationship from the feature space to the corresponding subjective scores (MOS/DMOS).
The statistics of NSS in spatial domain (SSD) or transform domains (STD) are investigated in different research studies. Mittal et al. ${ }^{7}$ proposed the BRISQUE method for BIQA by using NSS in spatial domain called mean subtracted contrast normalized (MSCN). Histograms of MSCN and their pairwise products are estimated by general Gaussian distribution (GGD) and asynchronous GGD, respectively. The parameters of the distributions are used to form a feature vector and a support vector regressor (SVR) is used to train the model using MOS/DMOS of the benchmark IQA databases. Wakeby distribution models proposed in Refs. 12 and 29 use Wakeby distribution, which is a better descriptor to model the MSCN coefficients and their pairwise products distributions. In this method, four parameters of Wakeby distribution including two shape parameters and two scale parameters are used to form a quality aware feature vector and then SVR is employed to model the complex nonlinear relationship from the feature space to the corresponding subjective scores.

In some other NSS-based BIQA methods, the statistics of transform domains (STD) are used. As an example, the NSS model based on DCT coefficients has been proposed by Saad et al. ${ }^{10}$ for BIQA. In this method, properties of DCT coefficients are used to form a quality-aware feature vector and then a Bayesian inference approach is utilized to predict the input images quality score. A method based on wavelet transform is introduced in Ref. 30 in which the wavelet coefficients are fitted by GGD and the parameters of the distribution are used to form a quality aware feature vector.

DIIVINE $^{9}$ is another BIQA method that utilizes NSS in the wavelet domain. This method is designed in two stages: 
first the distortion of the input image is predicted and then the quality score of the image is achieved by using the SVR model that trained in that distortion. He et al. ${ }^{31}$ used sparse representation of NSS features in the wavelet domain to propose a BIQA method. After extracting the NSS features, they form features via sparse coding and then obtain the quality of images by weighting DMOS by the sparse coding coefficients. Chu et al. ${ }^{32}$ presented a blind image quality metric based on quality aware features that are extracted from the joint distributions of neighboring divisive normalization transform coefficients over the space and scale. They used SVR to map the feature space to quality score of the input image. There are more algorithms for BIQA in the transform domain such as Curvelet domain ${ }^{33}$ and Shearlet domain. ${ }^{34}$ In another type of BIQA method, nonstatistical features (OIF) such as Gabor wavelet and gradients features are used to assess the input image quality. For example, Ye and Doermann ${ }^{35}$ extracted Gabor wavelet features from image blocks to form visual codebooks and then SVR is used to predict the quality score of the image. In Ref. 36, a method for BIQA is presented that uses phase congruency of images in order to form a quality-aware feature. They utilized a generalized regression neural network to predict the quality score.

The third subcategory of BIQA methods uses hybrids of NSS and OIF approaches. Wu et al. ${ }^{37}$ combined NSS features extracted from multiple domains and multiple color channels to form quality aware features for predicting the quality of an image. Universal BIQA that combines NSS features including non-Gaussianity, local dependency, and an exponential decay characteristic with multiple kernel learning has been proposed by Gao et al. ${ }^{38}$ in discrete wavelet transform domain.

The fourth group of BIQA methods uses DNN to assess the quality of the input image. Hou et al. ${ }^{39}$ proposed a DNNbased approach for BIQA. Their network learns from the linguistic description of image quality to classify the input image into five quality grades including excellent, good, fair, poor, and bad. Then, they used a pooling strategy to convert these classes to a continuous quality score.

As the large number of parameters should be trained in DNN, a huge number of training samples are needed to avoid overfitting. But the existing benchmark databases have a small number of samples. The DNN-based IQA methods use random patches from each image to overcome this limitation.

The last group of universal BIQA methods is based on all studied features (including NSS-based, non-NSS, or combined) but the methods are not learned by human opinion scores. Xue et al. ${ }^{40}$ used a percentile pooling strategy to estimate quality of overlapped blocks of an input image. Their clustering algorithm is trained by centroid blocks as a codebook to estimate the quality levels of the image blocks, then the scores of blocks are normalized, and the overall quality of the input image is inferred. Zhang et al. ${ }^{41}$ proposed a completely blind image quality method by integrating the features of NSS of neutral images derived from multiple domains; then their system learns a multivariate Gaussian model from each of the blocks from nondistorted pristine images. The quality of each image patch is estimated by measuring the deviation of the statistics of the patch from the reference statistics prelearned from high-quality natural images. The overall image quality score is then estimated by a pooling strategy.

Most of the BIQA methods assumed that the human visual system (HVS) considers images' distortion types and severities to estimate only objective scores, but the HVS also responds to image content and image aesthetics in subjective quality assessment. In this paper, we use a host of content-dependent features that are commonly employed in aesthetics assessment alongside the distortion aware features to improve the performance of the exiting BIQA methods.

\section{Proposed Features}

Many BIQA methods use NSS features and emulate subjective quality based on visible image distortions and neglect the influence of image content on BIQA. To fill this gap, we presented some aesthetics related measures in the fields of EA and photoquality assessment to estimate image quality. In this section, at first, all of these measures are listed and then the ones that are proposed for BIQA together with their proposed customization are described in detail. Table 1 summarizes the notations and symbols used in this paper, which may be useful in the rest of this paper.

\subsection{Review on Related Measures}

To consider image aesthetic features, we used some aesthetic features from the EA and photoquality assessment studies, then a feature selection method based on a genetic algorithm was utilized to select a subset of these features that have better correlation with human perceptual image quality assessment. In the EA works, we considered three measures proposed in Ref. 42, Machado and Cardoso, Ross, Ralph and Zong (bell curve), and a fractal dimension aesthetic measure. Also, we studied five measures proposed in Ref. 43 including Benford's law (first-digit law), Machado and Cardoso, image order, image complexity (IC), and color ingredient. In addition, the seven proposed features by den Heijer and Eiben ${ }^{44}$ were considered. These features are used to generate aesthetically pleasing images using an unsupervised evolutionary algorithm. These seven features are Benford's law, fractal dimension aesthetic measure, global contrast facto, information theory aesthetic measures, bell curve, and reflectional symmetry. Finally, measures for symmetry, compositional balance, and liveliness proposed in Ref. 45 are used. They used these features to compute a fitness function for an evolutionary algorithm to generate images with a good taste in HVS.

The photoquality assessment area of research, which tries to discriminate good photos from bad ones, uses aesthetic measures. Tang et al. ${ }^{46}$ proposed a method for content-based photoquality assessment that uses eight features including dark channel, clarity contrast, lighting contrast, composition geometry, IC, Hue composition, and scene composition. Yao et al. ${ }^{47}$ suggested OSCAR as a composition and aesthetic evaluation system for photography. This method is not only for discriminating good and bad photos, but also for rating them too. They used some visual features for black and white images, global and local sharpness, image tonality and contrast, convex hull and normalized inertia, image saliency, aspect ratio, and noise level for this aim. 
Table 1 Notations used in this paper and their relative meanings.

\begin{tabular}{|c|c|c|c|}
\hline Symbol & Meaning & Symbol & Meaning \\
\hline$d$ & First digit in Benford's distribution & $\alpha$ & Shape in GGD fitting \\
\hline$P$ & Probability in Benford's distribution & $\rho$ & Dependent shape parameter in GGD \\
\hline r & Vector containing all $P$ numbers & $\sigma^{2}$ & Variance \\
\hline$x$ & Vector of subject's first digits & $x$ & Hue histogram of image \\
\hline$C$ & Coefficients for each digit & $\Gamma$ & Integration parameter in GGD \\
\hline$\theta$ & A function which returns the first digit & $s$ & Subject area \\
\hline$N$ & Number of self-similar pieces & $\lambda$ & Constant coefficient for angular width \\
\hline$\varsigma$ & Magnification factor & $A$ & Angular width of a template \\
\hline I & Image & $T_{k}$ & Color template of an image \\
\hline IC & Image complexity & $T$ & Set of all $T s$ \\
\hline PC & Processing complexity & $\tau_{k}$ & minimum distance between current image and $T_{k}$ \\
\hline$t$ & Time & $\tau_{k, i}$ & Minimum distance between current image and $T_{k, i}$ \\
\hline$w$ & Weight & $T_{k, i}$ & Color template of an image rotated by I \\
\hline$L$ & luminance of a pixel & $D$ & Arc-length function \\
\hline$C_{i}$ & Global contrast for resolution $i$ & $\beta$ & A bin of histogram \\
\hline$I c_{i}$ & Pixel contrast in resolution $i$ & & \\
\hline
\end{tabular}

Guo et al. ${ }^{48}$ proposed another method for image aesthetic assessment that uses handcrafting and semantic features. Also, a set of aesthetic features are proposed by $\mathrm{Li}$ and Chen ${ }^{49}$ These aesthetic features were extracted from both global characteristics and local characteristics of an image. These features represent the color, brightness, and composition concepts in a painting image.

To identify the most important aesthetic features in image quality and also to reduce the redundancy between the studied aesthetic features, we used a feature selection method. ${ }^{50}$ This method is designed to select the more informative features for image quality from all aesthetic features. The goal is to find an optimal subset of features by minimizing the number of used features and quality prediction error. The selected aesthetic features set has a significant correlation with human perceptual image quality and is also not redundant.

We ran the feature selection method in many IQA benchmark databases to select an informative subset of all features mentioned above from the EA and photoquality assessment. From all these features, the feature selection method selected 11 aesthetic features that perform the best image quality performance on the benchmark IQA databases. The selected features are as follows:
- Benford's law (first-digit law);

- fractal dimension aesthetic measure;

- Machado and Cardoso;

- image complexity (IC);

- processing complexity (PC);

- global contrast factor;

- Ross, Ralph, and Zong (bell curve);

- dark channel;

- colorfulness;

- hue composition;

- color harmony;

- color saturation.

Table 2 demonstrates the performance of a machine learner trained by each aesthetic feature. The performance is reported using linear correlation coefficient (LCC) between the predicted scores and the ground truth quality scores (MOS/DMOS) on the testing benchmark IQA databases. These databases are TID2013, ${ }^{14} \mathrm{CSIQ}^{51}{ }^{51}$ and LIVE ${ }^{52}$, which will be introduced in detail in Sec. 4. In the following, we discuss the implementation of these features. We also customized the implementation of some of them such as Benford's 
Table 2 LCC between each feature value and the MOS/DMOS on the testing IQA databases. All LCC values higher than 0.4 are bold.

\begin{tabular}{|c|c|c|c|c|c|c|c|c|c|c|c|c|}
\hline Database & Distortion & $f_{\mathrm{BL}}$ & $f_{\mathrm{FD}}$ & $f_{\mathrm{MC}}^{v 1}$ & $f_{\mathrm{MC}}^{\vee 2}$ & $f_{\mathrm{GCF}}$ & $f_{\mathrm{BC}_{1}}$ & $f_{\mathrm{BC}_{2}}$ & $f_{\mathrm{DC}}$ & $f_{\mathrm{CF}}$ & $f_{\mathrm{HC}_{1}}$ & $f_{\mathrm{HC}_{2}}$ \\
\hline \multirow[t]{24}{*}{ TID2013 } & Additive Gaussian noise & 0.021 & 0.2855 & 0.5051 & 0.6799 & 0.3871 & 0.4524 & 0.2467 & 0.281 & 0.4766 & 0.4118 & 0.2622 \\
\hline & Additive noise in color components & 0.0198 & 02954 & 0.5206 & 0.4816 & 0.4236 & 0.3885 & 0.3748 & 0.57 & 0.5467 & 0.4001 & 0.3026 \\
\hline & Spatially correlated noise & 0.035 & 0.3424 & 0.3534 & 0.4756 & 0.4927 & 0.3976 & 0.2158 & 0.4891 & 0.4497 & 0.4217 & 0.2626 \\
\hline & Masked noise & 0.1121 & 0.0328 & 0.1401 & 0.4409 & 0.0554 & 0.251 & 0.1775 & 0.2133 & 0.2109 & 0.1844 & 0.1326 \\
\hline & High-frequency noise & 0.0411 & 0.2467 & 0.6925 & 0.8461 & 0.5731 & 0.5742 & 0.0403 & 0.6445 & 0.741 & 0.5223 & 0.3272 \\
\hline & Impulse noise & 0.0332 & 0.208 & 0.5238 & 0.7565 & 0.4829 & 0.0711 & 0.0885 & 0.7936 & 0.872 & 0.2179 & 0.0841 \\
\hline & Quantization noise & 0.7031 & 0.2493 & 0.31 & 0.066 & 0.0105 & 0.0005 & 0.4325 & 0.0226 & 0.079 & 0.7426 & 0.6729 \\
\hline & Gaussian blur & 0.0247 & 0.0976 & 0.4962 & 0.5709 & 0.1918 & 0.0179 & 0.0002 & 0.7297 & 0.3191 & 0.0702 & 0.0457 \\
\hline & Image denoising & 0.1113 & 0.1735 & 0.4486 & 0.5563 & 0.3178 & 0.3168 & 0.4187 & 0.1765 & 0.4902 & 0.1421 & 0.0246 \\
\hline & JPEG compression & 0.2694 & 0.1363 & 0.0084 & 0.2236 & 0.0139 & 0.5726 & 0.6612 & 0.1352 & 0.1275 & 0.2653 & 0.0106 \\
\hline & JPEG2000 compression & 0.0058 & 0.0023 & 0.6347 & 0.7002 & 0.5749 & 0.2272 & 0.3625 & 0.6682 & 0.4897 & 0.1394 & 0.1671 \\
\hline & JPEG transmission errors & 0.0729 & 0.0137 & 0.2255 & 0.1306 & 0.1843 & 0.0042 & 0.0724 & 0.1892 & 0.1274 & 0.0839 & 0.0485 \\
\hline & JPEG2000 transmission errors & 0.0626 & 0.0057 & 0.061 & 0.2808 & 0.3269 & 0.0579 & 0.1265 & 0.3497 & 0.3051 & 0.1212 & 0.08 \\
\hline & Noneccentricity pattern noise & 0.026 & 0.0854 & 0.1083 & 0.0161 & 0.0607 & 0.0147 & 0.0623 & 0.0656 & 0.03 & 0.0976 & 0.1004 \\
\hline & Local block-wise distortions & 0.1233 & 0.0468 & 0.1494 & 0.2063 & 0.1962 & 0.0787 & 0.162 & 0.0342 & 0.0998 & 0.011 & 0.0136 \\
\hline & Mean shift (intensity shift) & 0.2533 & 0.0326 & 0.1793 & 0.2629 & 0.1664 & 0.2504 & 0.3151 & 0.3371 & 0.415 & 0.1693 & 0.0864 \\
\hline & Contrast change & 0.4612 & 0.4464 & 0.4927 & 0.3746 & 0.5655 & 0.1002 & 0.0928 & 0.8108 & 0.6788 & 0.5928 & 0.0283 \\
\hline & Change of color saturation & 0.1368 & 0.431 & 0.2848 & 0.4538 & 0.3641 & 0.2249 & 0.3096 & 0.3454 & 0.13 & 0.269 & 0.004 \\
\hline & Multiplicative Gaussian noise & 0.1239 & 0.0095 & 0.5187 & 0.6725 & 0.4844 & 0.3389 & 0.2353 & 0.154 & 0.4633 & 0.4331 & 0.221 \\
\hline & Comfort noise & 0.0457 & 0.071 & 0.1491 & 0.2335 & 0.1023 & 0.2659 & 0.2225 & 0.1755 & 0.0672 & 0.161 & 0.1288 \\
\hline & Lossy compression of noisy images & 0.0662 & 0.1471 & 0.0131 & 0.2382 & 0.1268 & 0.349 & 0.4459 & 0.1335 & 0.056 & 0.2042 & 0.1882 \\
\hline & Image color quantization with dither & 0.7866 & 0.5608 & 0.2589 & 0.546 & 0.153 & 0.5258 & 0.5573 & 0.4778 & 0.2282 & 0.6139 & 0.5386 \\
\hline & Chromatic aberrations & 0.2429 & 0.2223 & 0.6595 & 0.7069 & 0.6164 & 0.0391 & 0.001 & 0.7156 & 0.4436 & 0.2135 & 0.0834 \\
\hline & Sparse sampling and reconstruction & 0.0374 & 0.1353 & 0.6862 & 0.7191 & 0.5164 & 0.0687 & 0.1329 & 0.5445 & 0.4808 & 0.174 & 0.1134 \\
\hline \multirow[t]{6}{*}{ CSIQ } & Noise & 0.0269 & 0.0415 & 0.3144 & 0.6039 & 0.1556 & 0.3714 & 0.1442 & 0.2282 & 0.507 & 0.1401 & 0.1235 \\
\hline & JPEG & 0.4714 & 0.0521 & 0.2548 & 0.2321 & 0.2921 & 0.6159 & 0.6878 & 0.086 & 0.4373 & 0.5789 & 0.0247 \\
\hline & JPEG2000 & 0.1592 & 0.0246 & 0.6317 & 0.6589 & 0.5283 & 0.3218 & 0.3936 & 0.5403 & 0.4446 & 0.1371 & 0.121 \\
\hline & Fnoise & 0.0459 & 0.0997 & 0.1956 & 0.196 & 0.2167 & 0.3968 & 0.1295 & 0.2857 & 0.3622 & 0.1589 & 0.0851 \\
\hline & Blur & 0.0298 & 0.0664 & 0.7462 & 0.7382 & 0.6758 & 0.1586 & 0.0292 & 0.6113 & 0.5291 & 0.27 & 0.0552 \\
\hline & Contrast & 0.0672 & 0.4184 & 0.6441 & 0.3672 & 0.6168 & 0.2888 & 0.294 & 0.154 & 0.7767 & 0.6806 & 0.1141 \\
\hline \multirow[t]{5}{*}{ LIVE } & JPEG2000 & 0.0184 & 0.1372 & 0.1537 & 0.3687 & 0.0442 & 0.0998 & 0.0942 & 0.3737 & 0.125 & 0.0158 & 0.0733 \\
\hline & JPEG & 0.365 & 0.0298 & 0.0218 & 0.2058 & 0.0585 & 0.5534 & 0.7433 & 0.0096 & 0.2486 & 0.6337 & 0.047 \\
\hline & WN & 0.6677 & 0.1083 & 0.7609 & 0.8541 & 0.6909 & 0.0381 & 0.4999 & 0.7945 & 0.7279 & 0.2709 & 0.2119 \\
\hline & Gblur & 0.0755 & 0.0417 & 0.6355 & 0.7015 & 0.6089 & 0.1761 & 0.0784 & 0.5927 & 0.5323 & 0.1906 & 0.1992 \\
\hline & Fast fading & 0.0004 & 0.0526 & 0.5614 & 0.6647 & 0.5106 & 0.1419 & 0.085 & 0.6024 & 0.4425 & 0.0784 & 0.1832 \\
\hline
\end{tabular}


law, Machado and Cardoso, and PC for achieving a better performance in BIQA.

\subsection{Implementation of the Selected Features}

\subsubsection{Benford's law $\left(f_{B L}\right)$}

Simon Newcomb (1835 to 1909) believed that in calculations, numbers that start with lower digits occur more often than numbers with larger leading digits. He found Eq. (1) that matched his observations ${ }^{53}$

$P=\log _{10} \frac{d+1}{d}$

where $P$ is the percentage of numbers that start with the digit $d$ in calculation with decimal number representation. Newcomb never proved his equation. The physicist Frank Benford noticed the same pattern in 1938. He collected lots of data and showed this equation matches them pretty well. However, the proof remained an open problem until Roger Pinkham did it and claimed "if a law of digit frequencies is scale invariant then it has to be Benford's Law," but the law finally was named after Benford. ${ }^{53}$

Benford's law is valid if the data are not totally random nor overly constrained, but rather lie somewhere between. For example, random numbers generated by a computer (pseudo) random generator are totally random and the height of males in Tehran is considered overly constrained, but the numbers that appear on the first page of a newspaper throughout some time, lies between them and matches with Benford's law distribution for the first digit.

Some EA works like Refs. 43 and 44 apply Benford's law on images. An image is considered to be natural and visually appealing when the histogram of pixel luminance values obeys the natural equation of Benford's law [Eq. (1)]. We proposed a certain way to use Benford's law for image quality assessment. We created a histogram of luminance pixels in 256 bins then counted first digits of the histogram bins. The following equation calculates the distance between the perfect Benford's law distribution and the input images histogram. Let $f_{0}, \ldots f_{255}$ denote the frequencies of pixel gray scale into intensities $0, \ldots, 255$. Let $X_{d}, d=1, \ldots, 9$ denote the normalized number of frequencies $f_{i}$ that have the leading digit $d$, therefore

$$
F_{\mathrm{BL}}=\sum_{d=1}^{9} C_{d}\left|X_{d}-\log _{10} \frac{d+1}{d}\right|,
$$

where $C_{d}$ is coefficient for each digit. The $C$ coefficients are responsible for weighting the distance in Euclidean space. We employed an evolutionary strategy to find out which digit (from 1 to 9) is more important to Benford's law, then we found a set of coefficients for the digit deviations that had better correlation with MOS/DMOS scores. To do this, the problem has been converted into an optimization one for which the goal was to find the best LCC of Benford's measure with the MOS/DMOS of the distorted images of the databases.

Figure 3 shows that the statistics of the proposed Benford's law for image quality follow the Benford's law distribution for natural images. The blue bars represent Benford's actual distribution coefficients, whereas red bars are the mean histogram extracted in our method from all the reference images of the LIVE ${ }^{52}$ IQA database.

\subsubsection{Fractal dimension aesthetic measure $\left(f_{F D}\right)$}

The aesthetic preference of humans for several types of fractals is studied by Spehar et al. ${ }^{54}$ They investigated natural, artificial, and man-made fractal images to study image aesthetic appeal. This study shows a consistent trend for aesthetic preference to peak within the fractal dimension range 1.3 to 1.5 . Images with a higher fractal dimension were considered as complex images, and images with a lower dimension were considered uninteresting images and both have a low preference. Motivated by this outcome, den Heijer and Eiben ${ }^{44}$ defined a fractal dimension aesthetic measure $f_{\mathrm{FD}}$ for a given image $I$ with a Hausdorff fractal dimension $f_{\mathrm{HD}}$ as follows:

$f_{\mathrm{FD}}=\max \left[0,1-\left|1.35-f_{\mathrm{HD}}(I)\right|\right]$.

In order to compute the fractal dimension of an input image, a box counting technique ${ }^{54}$ has been employed, which is an approximation of Hausdorff dimension. Considering an

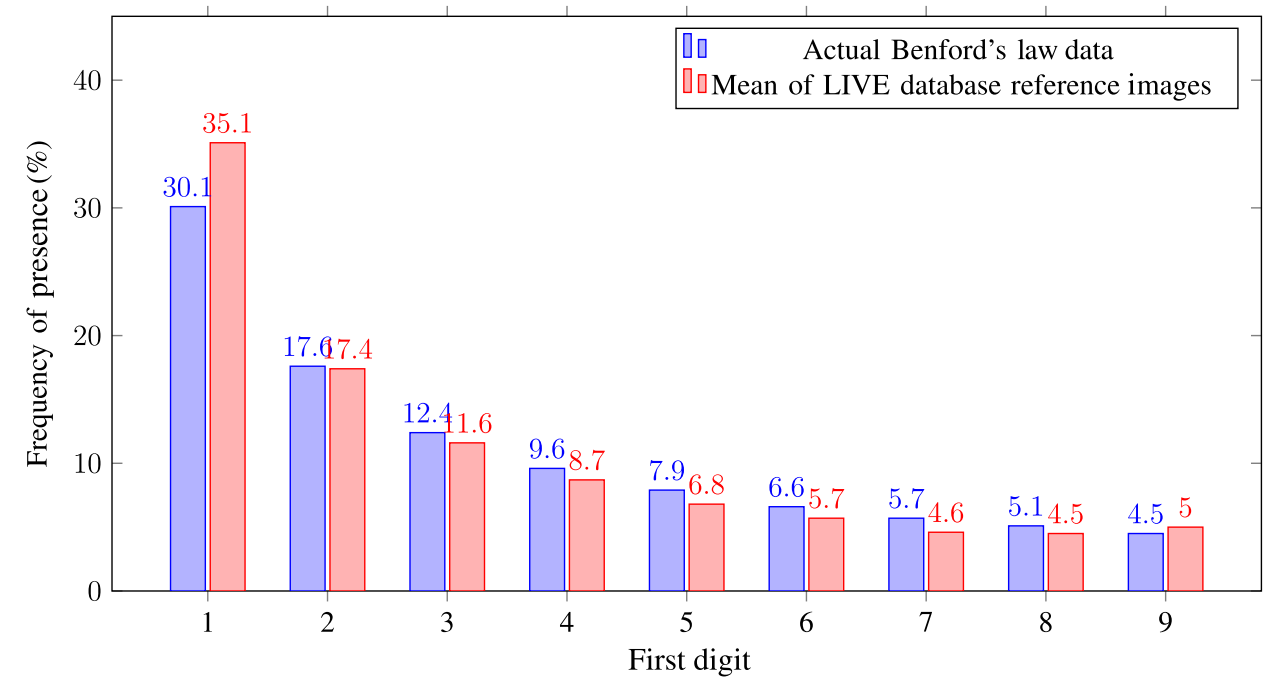

Fig. 3 The statistics of Benford's law for image quality follow the actual Benford's law statistics. The blue bars represent Benford actual distribution coefficients, whereas red bars are the mean value of the proposed method of all the reference images of LIVE IQA database. 
object that possesses a Euclidean dimension $E$, the Hausdorff fractal dimension $f_{\mathrm{HD}}$ can be computed by

$f_{\mathrm{HD}}=\lim _{x \rightarrow 0} \frac{\ln N(\varepsilon)}{\ln \varepsilon^{-1}}$

where $N(\varepsilon)$ is the counting of hyper-cubes of dimension $E$ and length $\epsilon$ that fills the object.

We used the fractal dimension aesthetic measure as one of our aesthetic features.

\subsubsection{Machado and Cardoso ( $f_{M C}^{v 1}$ and $\left.f_{M C}^{v 2}\right)$}

This EA native measure is based on the relation between IC and PC of a visual stimulus. ${ }^{55}$ Machado and Cardoso ${ }^{55}$ claimed that an image is more aesthetically pleasing when its visual complexity is high and it can be processed easily. Two implementations of this measure have been used as two features in our work.

The first implementation is the result of

$f_{\mathrm{MC}}^{v 1}=\frac{\mathrm{IC}(I)}{f_{\mathrm{HD}}(I)}$,

where IC is described in Eq. (6) and $f_{\mathrm{HD}}(I)$ is the Hausdorff dimension of the image and can be computed using Eq. (4)

$\mathrm{IC}(I)=\frac{\operatorname{RMSE}\left(I^{\prime}\right)}{\text { compression } \operatorname{ratio}\left(I, I^{\prime}\right)}$,

where $I^{\prime}$ is the compressed image using a technique like JPEG or a compression method based on singular value decomposition. ${ }^{56}$

The second approach to implement this feature is described in

$f_{\mathrm{MC}}^{v 2}=\frac{\mathrm{IC}(I)}{\mathrm{PC}(I)}$,

where IC $(I)$ can be computed from Eq. (6) and in order to compute PC $(I)$, the method proposed in Ref. 43 has been employed exactly. This method uses fractal image compression, in which the image varies in the perception process as time passes and measures $\mathrm{PC}(I)$ separately into two time points $t_{0}$ and $t_{1}$ and estimates the measure using

$\mathrm{PC}(I)=\left[\mathrm{PC}\left(t_{0}\right) \times \mathrm{PC}\left(t_{1}\right)\right] \times\left[\frac{\mathrm{PC}\left(t_{0}\right)-\mathrm{PC}\left(t_{1}\right)}{\mathrm{PC}\left(t_{1}\right)}\right]$.

In conclusion, both types of PC are implemented then two different kinds of Machado and Cardoso measures are computed $\left(f_{\mathrm{MC}}^{v 1}\right.$ and $\left.f_{\mathrm{MC}}^{v 2}\right)$, which both showed significant correlation with subjective quality.

\subsubsection{Global contrast factor $\left(f_{G C F}\right)$}

Images with little difference values in their contrast are considered not aesthetically pleasing. This measure computes luminance differences at various resolutions. ${ }^{57}$

We used the same implementation of Ref. 57, which suggests computation of the global contrast factor in nine different resolutions. Equation (9) demonstrates computing this feature
$f_{\mathrm{GCF}}=\sum_{i=1}^{9} w_{i} * C_{i}$

where $w_{i}$ is exactly like the vector in Ref. 57: $w_{i}=$ $\left(-0.406385 * \frac{i}{9}+0.334573\right) * \frac{i}{9}+0.0877526 \mid i \in\{1,2, \ldots, 9\}$ and $C_{i}$ is

$C_{i}=\frac{1}{w * h} * \sum_{i=1}^{w * h} l c_{i}$

where $l c_{i}$ is

$l c_{i}=\frac{\left|L_{i}-L_{i-1}\right|+\left|L_{i}-L_{i+1}\right|+\left|L_{i}-L_{i-w}\right|+\left|L_{i}-L_{i+w}\right|}{4}$,

where each $L$ represents a pixel luminance value in the image after applying gamma correction with $\gamma=2.2$ in order to get a wider field for contrasts.

\subsubsection{Bell curve (Ross, Ralph, and Zong) feature $\left(f_{B C_{1}}\right.$ and $\left.f_{B C_{2}}\right)$}

Bell curve is a measurement based on the observation that color shape distribution, regarding the implicit aesthetic ideal of many painters throughout history, ${ }^{58}$ is normal (bell-shape). For computing of this feature, the hue histogram of an image $\{\operatorname{hist}[H(I)\}$ is extracted. Then, after normalizing the histogram, we tried fitting a $\mathrm{GGD}^{59}$ on the histogram. GGD with zero mean is

$f\left(x, \alpha, \sigma^{2}\right)=\frac{\alpha}{2 \rho \Gamma(1 / \alpha)} \exp \left[-\left(\frac{|x|}{\rho}\right)^{\alpha}\right]$,

where $\rho$ is computed using

$\rho=\sigma \sqrt{\frac{\Gamma(1 / \alpha)}{\Gamma(3 / \alpha)}}$,

and $\Gamma$ is a function described by

$\Gamma(a)=\int_{0}^{\infty} t^{a-1} e^{-t} \mathrm{~d} t a>0$.

In GGD, $\alpha$ is named the shape parameter and $\sigma^{2}$ is variance of the distribution. These two parameters for hue histogram are employed as our bell curve feature $\left(f_{\mathrm{BC}_{1}}\right.$ and $f_{\mathrm{BC}_{2}}$, respectively).

\subsubsection{Dark channel $\left(f_{D C}\right)$}

The dark channel is designed for image haze removal. The intensity of some pixels (called "dark pixels") of the haze free images, have very low intensity in at least one of the Red, Green or Blue channels and have higher intensity in regions with denser haze. Therefore, these dark pixels can directly provide an accurate estimation of the haze's transmission. ${ }^{60}$ Equation (15) shows the definition of this measure

$I_{\mathrm{dark}}(i)=\min _{c \in R, G, B}\left[\min _{i^{\prime} \in \Omega(i)} I_{c}\left(i^{\prime}\right)\right]$, 
where $I_{c}$ is a color channel of the image $I$ (red, green, and blue) and $\Omega(i)$ is a function returning a set of neighbor pixels of $i$. We defined the neighborhood as a local patch of $10 \times 10$. The dark channel feature of the image $I$ is computed by

$f_{\mathrm{DC}}=\frac{1}{\|S\|} \sum_{(i) \in S} \frac{I_{\mathrm{dark}}(i)}{\sum_{c \in R, G, B} I_{c}(i)}$,

where $S$ is the area of the image that the feature is extracting. The original method computes in whole images. For achieving better correlation with image quality, first a method to separate the input image foreground is applied then the foreground area is used as the $S$ set. In this implementation, the method in Ref. 61 was applied to the image in order to separate foreground from the background.

\subsubsection{Colorfulness $\left(f_{C F}\right)$}

The colorfulness metric is computed using the method proposed by Hasler and Suesstrun. ${ }^{62}$ With the RGB channels of a frame as matrices $\mathrm{R}, \mathrm{G}$, and $\mathrm{B}$, one computes two matrices $\mathrm{rg}=\mathrm{R}-\mathrm{G}$ and $\mathrm{yb}=\frac{1}{2}(\mathrm{R}+\mathrm{G})-\mathrm{B}$. Then, the metric is computed as

$f_{\mathrm{CF}}=\sqrt{\sigma_{\mathrm{rg}}^{2}+\sigma_{\mathrm{yb}}^{2}}+\frac{3}{10} \sqrt{\mu_{\mathrm{rg}}^{2}+\mu_{\mathrm{yb}}^{2}}$

where $\sigma_{\text {. }}^{2}$ and $\mu$. denote the variance and mean of the values in their respective matrices. Finally, the average value over all frames yields the colorfulness metric of a video.

\subsubsection{Hue composition $\left(f_{H_{C_{1}}}\right.$ and $f_{H C_{2}}$ )}

An appropriate arrangement of colors in a photograph can please the viewers and make a sense of harmony and order. In photography, after taking a picture, an expert photographer tries to adjust the hue composition with common color templates. It makes it more aesthetically pleasing. Figure 4 shows the common color template taken from the art of photography. ${ }^{63}$
The procedure of computing this measure of aesthetics is as follows.

The Harmonic templates $T_{k}$ on the hue wheel shown in Fig. 4 define the set $T$. If the given image $I$ is a gray scale picture, it will be considered that the image matches with the template of $N$ type and the algorithm is done; otherwise, we compute the distance between each template and the angular hue histogram of the image. The template with the minimum distance was selected as the best matching template. The total angular distance for the best matching template is taken as the feature for the algorithm. Equation (18) shows how the hue composition feature could be computed.

$\tau_{k}=\operatorname{argmin}_{i=0}^{2 \pi}\left(\tau_{k, i}\right)$,

where $\tau_{k, i}$ is

$\tau_{k, i}=\sum_{\beta \in B} H(\beta) *\left\{D\left[T_{k, i}(\beta)\right] * S(\beta)+\lambda A\left(T_{k}\right)\right\}$,

where $B$ is the set of all bins in the angular hue histogram of the image and $H(\beta)$ returns the number of pixels yield in the bin $\beta$ of the histogram. $S(\beta)$ is the mean saturation of the pixels in the $\beta$ 'th bin of the angular hue histogram and $\lambda$ is a constant equal to 0.03 . $A\left(T_{k}\right)$ returns the total angular width of template $T_{k}$ and $D$ is the arc-length function described as follows:

$D\left[T_{k, i}(\beta)\right]=\left\{\begin{array}{cc}0 & \beta \in T_{k, i} \\ \left\|\beta-T_{k, i}\right\| & \beta \notin T_{k, i}\end{array}\right.$,

where $\beta$ is considered being in $T_{k, i}$ when the template $T_{k}$ is rotated by the angle $i$ and $\beta$ falls in one of its sectors. $\left\|\beta-T_{k, i}\right\|$ returns the arc-length distance between $\beta$ nearest sector border of $T_{k, i}$. If image $I$ is fitted with a single-sector template, the average saturation of pixels inside this sector $S(\beta)$ and the hue center of the fitting sector $\alpha$ are used as the hue composition features $f_{\mathrm{HC}_{1}}$ and $f_{\mathrm{HC}_{2}}$ respectively. If the image $I$ is fitted with a two-sector template, then the average saturations and hue centers are used as the hue composition features. $^{46}$
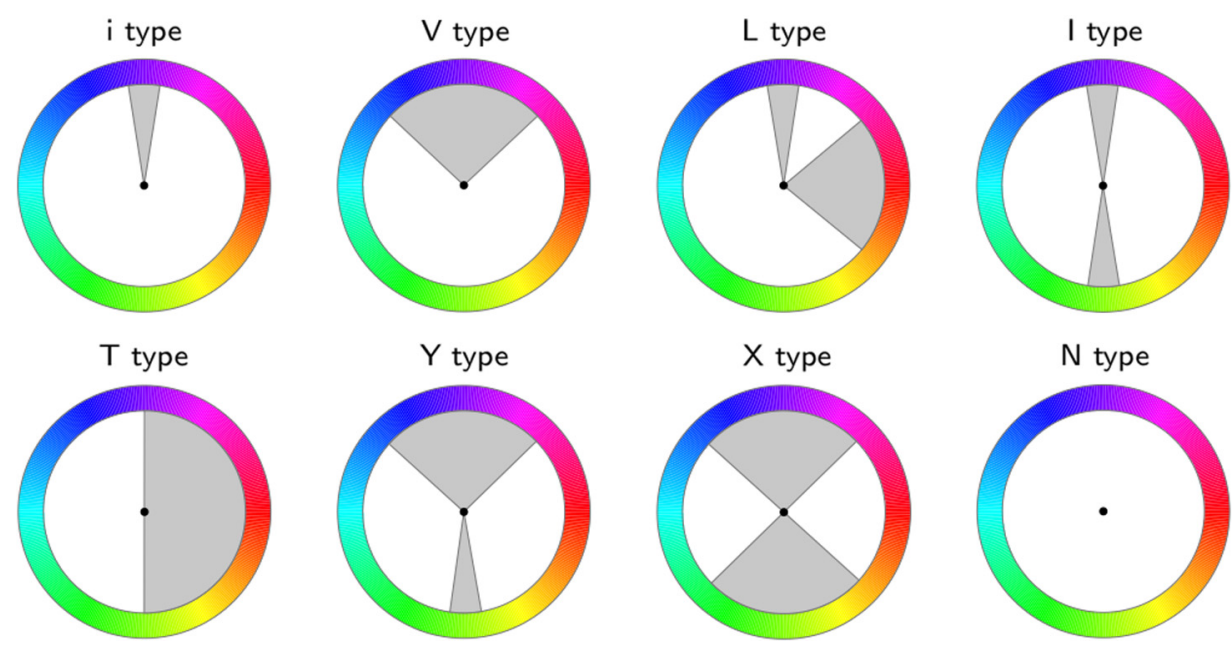

Fig. 4 Hue color wheel templates from Ref. 63. Images which most of their hue fall into gray areas of these templates are considered harmonic. Templates could be rotated by any angle. 
In the next section, we used the proposed aesthetic features to enrich five state-of-the-art BIQA methods. Then, we conducted experiments to study their performance improvements to predict subjective scores when their features are augmented by the proposed aesthetic features.

\section{Experimental Results}

In this section, first we discuss the image quality databases that are used in our experiments and their corresponding psychometric experiments for subjective quality assessment. Then, we conducted extensive experimental results to show how the proposed set of aesthetic features could improve the performance of some of the existing state-of-the-art objective BIQA methods.

\subsection{Datasets}

There are several datasets that have been established and made public for performance evaluation of IQA metrics. In the following, we listed the datasets used in our experiments and their characteristics.

- LIVE $^{52}$ contains 29 reference images and 779 distorted images in five types of distortion: JPEG2000, JPEG, white noise (WN) in RGB components, Gaussian blur in RGB components, and bit errors in JPEG2000 bit stream when transmitted over a stimulated Rayleigh channel. For collecting the subjective scores, about 20 to 29 human observers (subjects) rated each image from "bad" to "excellent" in five degrees. DMOS score is then calculated for each distorted image.

- CSIQ $^{51}$ consisting of 30 reference images attacked by six types of distortion in four or five different levels. Thirty-five human observers rated the images and the subjective scores have been reported in the form of DMOS.

- IVC $^{64}$ database consisting of 235 distorted images were generated from four different types of distortion from 10 reference images. The distortions included JPEG, JPEG2000, and LAR coding. Subjective evaluations were made at a viewing distance of six times the screen height using the double stimulus impairment scale method. ${ }^{65}$ Fifteen subjects evaluated each image and MOS values have been reported.

- Toyama ${ }^{66}$ the MICT Lab at the University of Toyama conducted subjective experiments to obtain subjects' quality scores for a set of images distorted with JPEG and JPEG2000 codec. Ninety-eight images were distorted by JPEG2000 and 98 other images were distorted by JPEG compression. This database is also known as MICT or Toyama-MICT image quality database.

- TID2008. ${ }^{67}$ TID stands for Tampere image database. Its arrival was for the sake of full-reference IQA but it is useful for no-reference as well. This database contains 25 reference images and 1700 distorted images. Each reference had been attacked by 17 distortions with 4 different levels each and that makes the number 1700. To collect the subjective scores, 838 observers from three countries (Finland, Italy, and Ukraine) rated the distorted images in double stimulus manner. Finally, MOS scores are reported with the database in range 0 (worst) to 9 (best).
- $\mathrm{TID} 2013^{14}$ is the successor of the TID2008 database and contains 25 reference images and has 24 types of distortions. The images have been attacked on five different levels. There are 3000 distorted images in the database $(25$ reference images $\times 24$ types distortions $\times 5$ levels of distortions) and MOS score for each image computed using 971 subjects from five different countries (Finland, France, Italy, Ukraine, and USA).

- MDID $^{68}$ consisting of 20 reference images and 1600 distorted images. Random types and levels of distortions are applied for generating distorted images from the reference images. Then, each distorted image contains five types of distortions simultaneously: Gaussian blur, contrast change, JPEG, JPEG2000, and Gaussian noise. According to the characteristics of image acquisition, transmission, and display of digital images, they applied the distortions as follows: Gaussian blur or contrast change first and JPEG and JPEG2000 second and then Gaussian noise.

\subsection{Psychometric Studies Analysis}

The subjective quality scores reported in the benchmark image quality databases are based on asking users to report their perceptual satisfaction with respect to a set of given images. Subjective ratings are often collected via psychometric experiments ${ }^{69}$ and aim at measuring the satisfaction of average users with respect to a given image in specific viewing conditions.

The human satisfaction may change with image degradation types and severities.

Many research studies in the fields of visual psychophysics have quantified relationships between pixel changes in visual stimuli like images and the corresponding psychological and neurophysiological responses. ${ }^{70-72}$

Chandler $^{73}$ studied the basic HVS properties that are explicitly or implicitly used in the existing IQA methods and he discussed some challenges that were chosen to highlight some limitations of the existing IQA methods in predicting human perceptual image quality. These limitations consist of HVS models for natural images, effects of distortions on image appearance, multiple types of distortions, geometric changes, enhanced images, and aesthetic quality.

Research in visual psychophysics aims to provide a better understanding of the HVS by linking changes in the physical attributes of a visual stimulus to the corresponding changes in psychological responses (visual perception and cognition). These studies generally involve carefully designed experiments on human subjects using highly controlled visual stimuli and viewing conditions.

All the IQA databases used in this paper to study the performance of BIQA methods have been obtained from the results of such studies. Table 3 summarizes the conditions and methods of psychometric evaluation of the seven databases that we used in our experimental evaluation.

The achieved MOS or DMOS from the corresponding subjective quality evaluations could be affected by the image content, the methodology of the subjective experiments, viewing condition, subject distance from the stimuli, number of subjects that evaluate each stimuli, etc. Figure 5 shows a normalized histogram of MOS/DMOS of the seven databases used in this paper. 
Table 3 Comparison of subjective evaluation conditions of seven benchmark image quality databases used in this paper.

\begin{tabular}{|c|c|c|c|c|c|c|c|}
\hline Databases & LIVE & CSIQ & IVC & Toyama & TID2008 & TID2013 & MDID \\
\hline Type of database & SD & SD & SD & SD & SD & MD & MD \\
\hline Number of reference image & 29 & 30 & 10 & 14 & 25 & 25 & 20 \\
\hline Number of distorted images & 779 & 866 & 185 & 196 & 1700 & 3000 & 1600 \\
\hline Number of types of distortion & 5 & 6 & 4 & 2 & 17 & 24 & 5 \\
\hline Distortion levels & 5 & $4-5$ & 4 & 6 & 4 & 5 & 4 \\
\hline Number of distortions per an image & 1 & 1 & 1 & 1 & 1 & $1-2$ & $1-4$ \\
\hline Methodology of subjective evaluation & SS & DS & DS & SS & $\mathrm{PC}$ & $\mathrm{PC}$ & PCR \\
\hline Screen & $21 " \mathrm{CRT}$ & 24"LCD & CRT & $17 " \mathrm{CRT}$ & 19"LCD & 19”LCD & $19 ” L C D$ \\
\hline Resolution & $\sim 768 \times 512$ & $512 \times 512$ & $512 \times 512$ & $768 \times 512$ & $512 \times 384$ & $512 \times 384$ & $512 \times 384$ \\
\hline Viewing distance & 2 to $2.5 S_{h}$ & $80 \mathrm{~cm}$ & $6 S_{h}$ & $4 H_{p}$ & Varied & Varied & $2 S_{h}$ \\
\hline Number of subjects & 29 & 25 & 15 & 16 & 839 & 971 & 192 \\
\hline Number of subjects rating per an image & 23 & 5 to 7 & 15 & 16 & 33 & 30 & 33 to 35 \\
\hline Scores format & $\mathrm{DMOS}+\delta$ & $\mathrm{DMOS}+\delta$ & MOS & $\operatorname{MOS}+\delta$ & $\mathrm{MOS}+\delta$ & $\mathrm{MOS}+\delta$ & $\mathrm{MOS}+\delta$ \\
\hline Scale of obtained estimates of scores & 1 to 100 & 0 to 1 & 1 to 5 & 1 to 5 & 0 to 9 & 0 to 9 & 0 to 8 \\
\hline Year of publication & 2006 & 2010 & 2006 & 2011 & 2009 & 2015 & 2017 \\
\hline
\end{tabular}

Note: SD, single distortion; MD, multiple distortions; SS, single stimuli; DS, double stimulus impairment scale; PC, pair comparison; PCR, pair comparison rating; $S_{h}$, height of viewing screen; $H_{p}$, height of the stimuli (e.g., image); MOS, mean opinion scores; DMOS, differential mean opinion scorers.

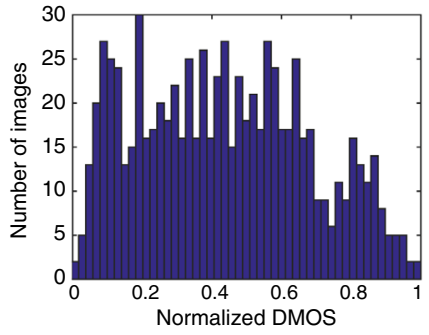

(a)

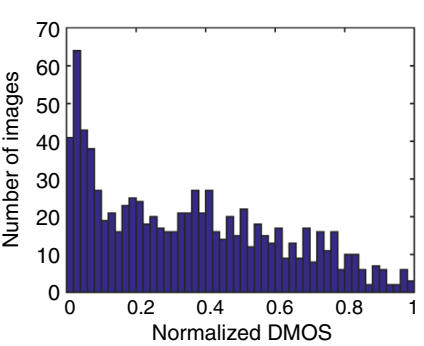

(e)

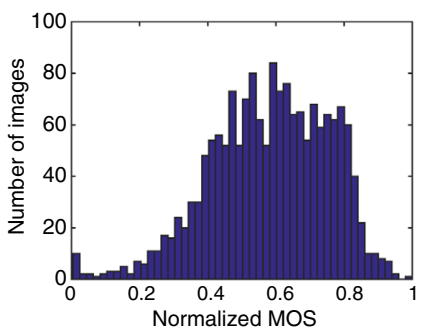

(b)

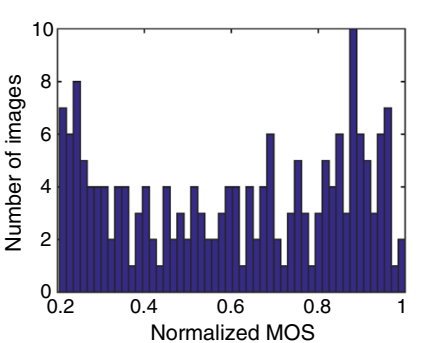

(f)

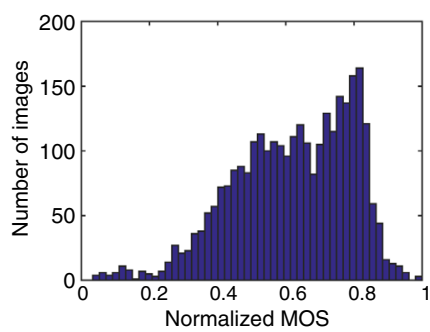

(c)

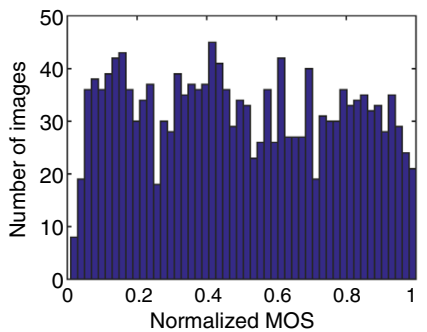

(d)

Fig. 5 Scatter plots of the histograms of the normalized MOSs/DMOSs for seven IQA databases used to evaluate the BIQA methods in this paper. (a) LIVE, (b) TID2008, (c) TID2013, (d) MDID, (e) CSIQ, (f) IVC, and $(\mathrm{g})$ Toyama. The MOSs/DMOSs values are normalized for better visualization. 
To show the subjects' agreement in the subjective evaluation of each database, we plotted the normalized standard deviations of the MOS/DMOS of the databases. In four databases including LIVE, TID2008, TID2013, and MDID, the normalized standard deviations of the scores over an image are less than 0.05. Figure 6 shows the normalized histogram of the standard deviations of MOS/DMOS for these databases. We also showed the median and mean of the standard deviations for better visualization.

In some of other IQA databases including CSIQ, IVC, and Toyama, the normalized standard deviations of MOS/ DMOS are much higher and near to 0.4. Figure 7 shows plots of the normalized standard deviations of these databases. One possible reason for this high variety of the standard deviations of the subjects' scores for the CSIQ database is the small number of subject rates per an image compared to other databases. As can be seen in Table 3, in CSIQ only 5 to 7 subjects rated each image where this number is 23 for the LIVE database.

The intergroup correlation of MOS/DMOS shows how well each of two separate groups of humans might be at predicting the response of the other. In the existing benchmark IQA databases used in this paper, only Toyama provides the raw quality scores per an image per each subject. Then, we can compute the intergroup correlation between two groups of subjects that rate the Toyama images. Figure 8 shows the correlation between two groups of eight people for rating Toyama images. We divided randomly the 16 subjects into two groups of 8 subjects, then computed the Spearman's rank ordered correlation coefficient (SROCC) between MOSs of these groups. This procedure was repeated 100 times to report the median SROCC between these two groups, which was 0.9685 .

It is worth mentioning that the max normalized standard deviation of the Toyama database is 0.2 , which is higher than standard deviations of LIVE, TID2013, TID2013, and MDID databases (see Figs. 6 and 7).

The intergroup correlation would present a fundamental limit beyond the correlation between the predicted quality scores by IQA methods and the subjective scores. Correlation improvements higher than the intergroup correlation between the subjects may show overfitting rather than real improvements, because the objective methods cannot predict the quality of an input image better than MOSs of another group of subjects.

\subsection{Aesthetic Features Enrichment Procedure}

In the following, first, we described the feature enrichment procedure of five state-of-the-art BIQA methods with the proposed aesthetic features. Then, we discussed the methodology of training and testing the BIQA method on the benchmark IQA database.

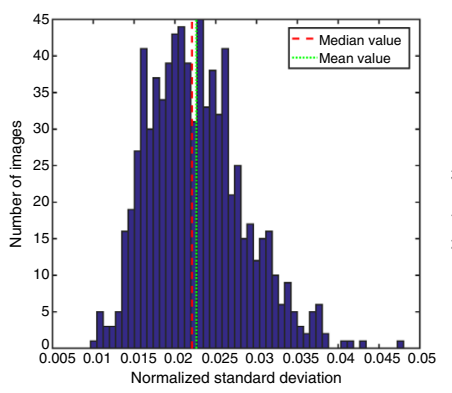

(a)

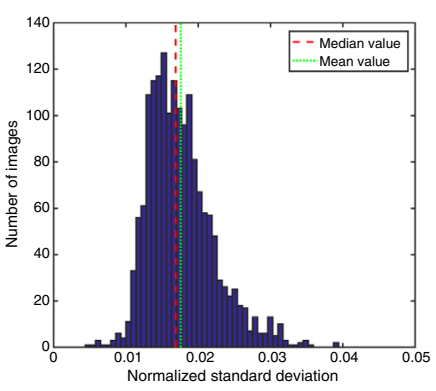

(b)

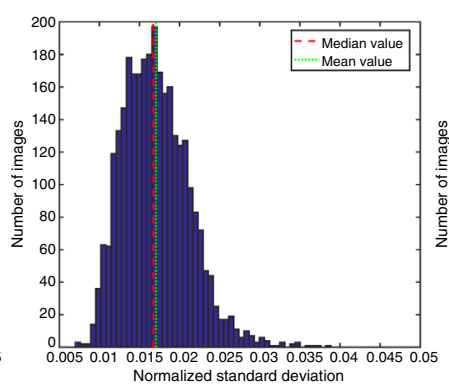

(c)

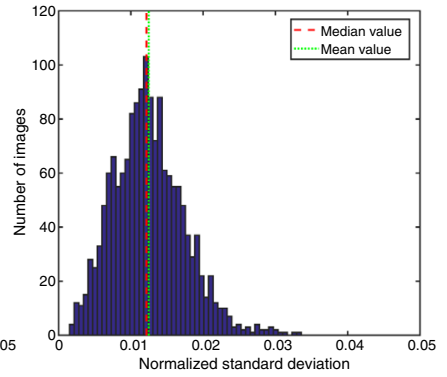

(d)

Fig. 6 Scatter plots of the histogram of normalized standard deviation of MOSs/DMOSs of four state-of-the-art databases. (a) LIVE, (b) TID2008, (c) TID2013, and (d) MDID. The standard deviations are normalized for better visualization. The maximum normalized standard deviation value in these databases is less than 0.05 .

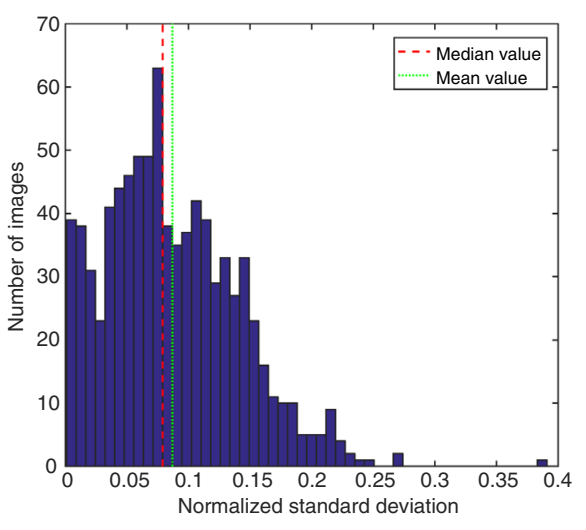

(a)

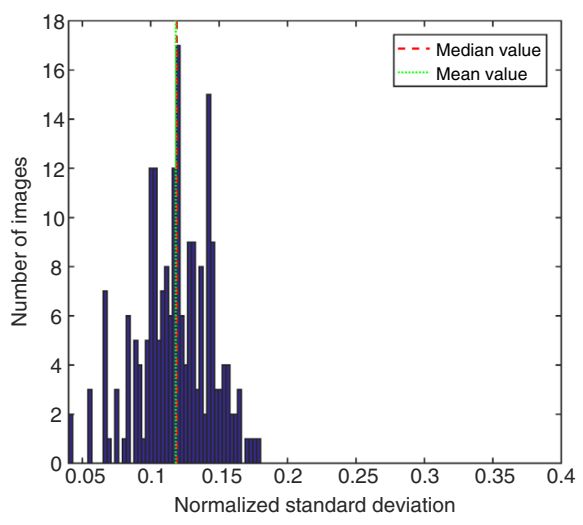

(b)

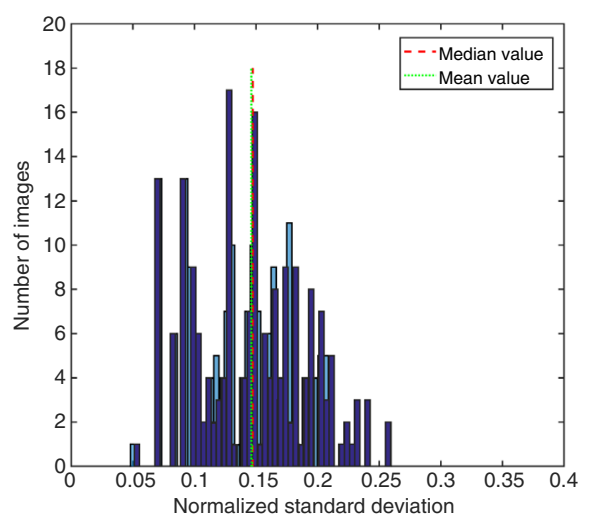

(c)

Fig. 7 Scatter plots of the histogram of normalized standard deviation of MOSs/DMOSs for three IQA databases. (a) CSIQ, (b) IVC, and (c) Toyama. The standard deviations are normalized for better visualization. The maximum normalized standard deviation value in these databases is around than 0.4 . 


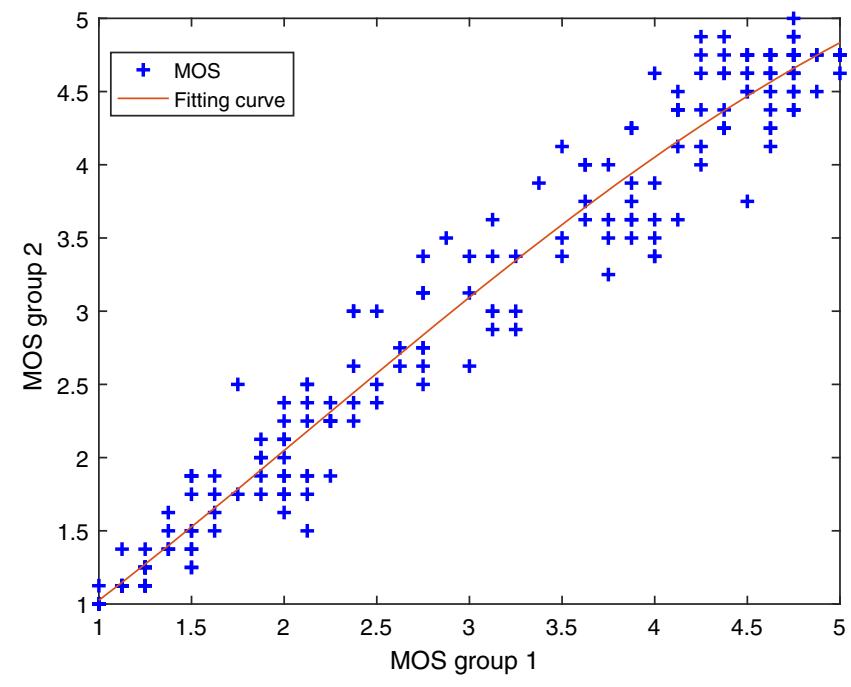

Fig. 8 Intergroup correlation between 16 subjects rated Toyama database images. We divided randomly the 16 subjects to two groups of 8 subjects and then MOSs of each group is computed.

\subsubsection{Enriching BIQA methods}

The proposed 11 aesthetic features described in Sec. 3.2 are introduced to help other methods in order to get better performance to estimate the subjective image quality scores. The feature enriching procedure for augmentation of the state-of-the-art BIQA features is described in the following.

Consider $M(\chi)$ as a learner regression machine that gets a feature vector $\chi$ in its input. Thus, a BIQA method $\Phi(I)$, which has a training phase on distorted images $(I)$, could be defined by

$\Phi(I)=M[\Omega(I)]$

where $\Omega(I)$ is a hypothetical function getting an image $I$ and returning a feature vector $\Psi$. Now, we can define $\Delta(I)$ as the function that in return of inputting an image gives back the vector of our proposed features $F$. Now $\Phi$, the BIQA method can be converted to $\Phi^{\prime}$ by putting vector $F$ beside $\Psi$ in both train and test phases

$\Phi^{\prime}(I)=M[\Omega(I)+\Delta(I)]=M(\Psi+F)$,

where + is an operator, which concatenates two vectors of its inputs. The process of making $\Phi^{\prime}$ is called enriching the method $\Phi$. As long as our work is focused on features only, we will utilize $M$ from each method for its own experiments and use the same $M$ after enriching the method. In the state-of-the-art methods that we employed to enrich, the authors used SVR as the regression machine. ${ }^{7,9,10,11,74} \mathrm{We}$ used the LIBSVM package ${ }^{75}$ implementation of SVR with the suggested parameters from each method.

\subsubsection{Testing on databases}

Let us denote all the distorted images of a database as $X$. Partition $X$ randomly into two subsets-80\% for training set $T$ and $20 \%$ for testing set $Z$ with the following conditions: $T \cup Z=X$ and $T \cap Z=\varnothing$. Now, assuming $|A|$ returns the total number of set $A$, we will have Eq. (23) with an $\alpha=0.8$ to obtain our wanted sets of $T$ and $Z$
$|T|=\alpha|X| \Leftrightarrow|Z|=(1-\alpha)|X|$

Now that definitions of $T$ and $Z$ are precisely described, we can see how our experiments held on the databases. In the following, we conducted extensive experimental results to show the effect of the proposed aesthetic features on the BIQA performance using benchmark image quality databases.

For each database (LIVE, CSIQ, IVC, Toyama, TID2008, TID2013, and MDID), we used $T$ and $Z$ as training and testing subsets for examining the performance of the state-of-the-art BIQA methods: BRISQUE, ${ }^{7}$ SSEQ $,{ }^{74} \mathrm{M} 3,{ }^{11}$ DIIVINE, ${ }^{9}$ and BLIINDS-II. ${ }^{10}$ Then, we concatenated the proposed aesthetic features to the feature vectors of each BIQA method and trained it on the same exact subset $T$ from the last experiment, thereafter all of them tested with $Z$. We repeated the steps 1000 times. The median value of correlation coefficient values of each method for each database is reported for performance comparison. Algorithm 1 shows the pseudocode of the performance evaluation for one database containing set $X$ of images.

Algorithm 1 Pseudocode for the performance evaluation of the enriched BIQA methods.

1. Input: $\mathbf{X}$ : image quality database with set $X$ of images, MOS: subjective quality scores of database $X, \Phi$ : set of BIQA methods, $\Omega($.$) : feature extractor function of each BIQA$ method, $\Delta($.$) : the proposed aesthetic feature vector$ extractor.

2. Output: $P$ : median SROCC/LCC value for original BIQA method, $P^{\prime}$ : median SROCC/LCC value for the enriched BIQA method on database $X$.

3. for all $\Phi$ such that $\Phi \in$ selected algorithms do

4. for $i=1$ to 1000 do

5. Partition $X$ into $T_{i}, Z_{i}$

6. $\operatorname{MOS}^{T} \leftarrow$ MOS of $T_{i}$

7. $\operatorname{MOS}^{Z} \leftarrow$ MOS of $Z_{i}$

8. $\Psi^{T} \leftarrow \Omega\left(T_{i}\right), F^{T} \leftarrow \Delta\left(T_{i}\right)$

9. $M \leftarrow$ train $M$ on $\Psi^{T}, M^{\prime} \leftarrow$ train $M$ on $\Psi^{T}+F^{T}$

10. $\Psi^{Z} \leftarrow \Omega\left(Z_{i}\right), F^{Z} \leftarrow \Delta\left(Z_{i}\right)$

11. $R \leftarrow M\left(\Psi^{Z}\right), R^{\prime} \leftarrow M^{\prime}\left(\Psi^{Z}+F^{Z}\right)$

12. $C_{i} \leftarrow \operatorname{correlation}\left(R, \operatorname{MOS}^{z}\right)$

13. $C_{i}^{\prime} \leftarrow$ correlation $\left(R^{\prime}, \operatorname{MOS}^{Z}\right)$

14. end for

15. $P \leftarrow \operatorname{median}(C)$

16. $P^{\prime} \leftarrow \operatorname{median}\left(C^{\prime}\right)$

17. end for 
To illustrate the performance improvement of the augmented BIQA by the proposed aesthetic features, two very common performance metrics were employed. One is the Pearson product-moment correlation coefficient also known as LCC which is the most common correlation measure and assesses the strength of the linear association between the predicted quality scores by a BIQA method and corresponding subjective quality scores. The other metric is SROCC which measures the monotonicity of the predicted quality scores. It is worth mentioning that the SROCC measuring the prediction monotonicity can be reliable even if the distribution of the data makes LCC undesirable or misleading, or different databases have a different MOS/DMOS scales.

\subsection{Aesthetics Feature Justification}

In this section, we conducted two different experiments to support the argument that any observed improvements in enriched BIQA methods are due to the inclusion of the proposed aesthetic features.

\subsubsection{Optimal feature selection analysis}

To justify the relevancy of the proposed aesthetic features in the performance improvements of the enriched BIQA methods, we conducted a feature selection experiment to study if the proposed aesthetic features will be selected by the feature selection algorithm as informative features for image quality or not. For this aim, we concatenated BRISQUE method features (36 dimensions) with the proposed aesthetic features (11 dimensions). Then, the enriched BRISQUE method by the aesthetic features has 47 dimension feature vectors.

Then, we utilized the NSGA-II feature selection method ${ }^{50}$ to select the more informative features for image quality from the augmented feature vector. This method is designed to find an optimal subset of features by minimization of both the number of used features and the classification error using the 1-NN classifier. In our experiments, we divided the MDID database to $60 \%$ train, $20 \%$ validation, and $20 \%$ test datasets. Also, we modified the feature selection method. We used support vector machine regressors for computing the cost function instead of the 1-NN classifier. Then, the median SROCC value of 1000 train-validation trial is used as the cost function.

The goal is to find the optimal subset of features that maximizes the SROCC for the validation dataset and then we used this subset of features to train a machine learner to predict the quality of the test set images. The experiments show this feature selection approach selects 32 features from the total 47 features where 8 of 11 aesthetic features were selected in the optimal subset.

This shows that the proposed aesthetic features are informative for augmenting the NSS-based features of the original method (BRISQUE) for the MDID database. We achieved 0.9210 SROCC value for the MDID database using this feature selection approach when the selected feature vector for the MDID database has 32 dimensions (includes 8 aesthetic features) where the performance of the original BRISQUE method is 0.8855 in this experiment. This experiment shows that the aesthetic features are informative for blind image quality and they make performance improvements even if the feature vector dimension of the enriched method is smaller (by using feature selection) than the original BIQA method.

\subsubsection{Principal component analysis}

To support the argument that any observed improvements in enriched BIQA methods are due to the aesthetic features enrichment and not because of increasing the feature dimension, in addition to the feature selection study, experiments based on PCA are conducted.

We computed the principal components of both the original and the augmented BRISQUE features for the challenging multiple distortion MDID image quality database. Then, we divided the database into $80 \%$ training set and $20 \%$ testing set. We trained a machine learner using only the first component as feature vector for both the original BRISQUE and the augmented version. Then, the SROCC metric for the predicted quality scores of the test set images and their corresponding subjective scores is computed. We repeated this experiment for the first two principal components until 36 first principal components. Figure 9 shows the performance of the original BRISQUE BIQA method and the enriched version for different feature dimensions using the most important PCA components from 1 to 36 components on the MDID database.

The experiment shows that the aesthetic features have a significant influence on the first principal components and they are informative for image quality. With the same feature dimension, the enriched method shows better performance for predicting the image quality scores.

\subsection{Performance Evaluation}

In this section, we conducted extensive experiments to illustrate the performance improvements of the tested BIQA methods when they are enriched by the proposed aesthetic features.

Table 4 shows the correlation between the predicted scores and the ground truth scores for the original version of some BIQA methods consisting of BRISQUE, SSEQ, M3, DIIVINE, and BLIINDS-II and their enriched versions. In this table, the results of the median LCC values for 1000 times repetition on the benchmark databases have been reported. The best accuracy in each row is bold and italic,

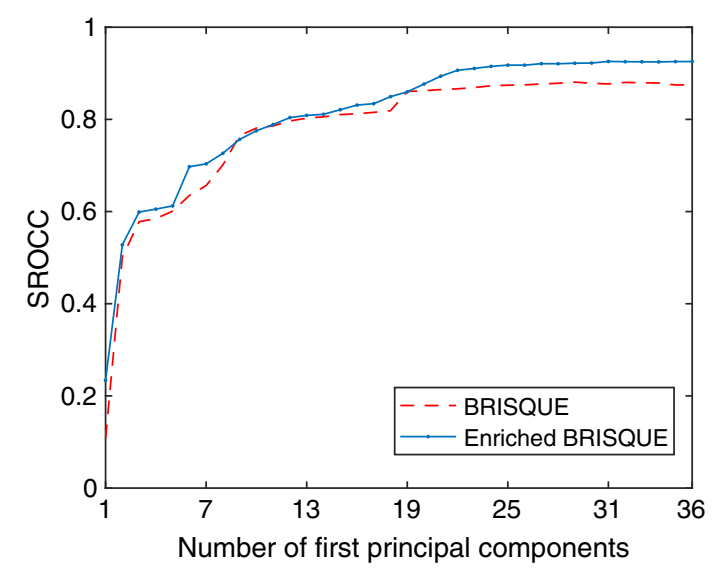

Fig. 9 The SROCC performance metric of the original BRISQUE BIQA method and its enriched version for different feature dimensions using most important PCA components from 1 to 36 components on MDID database. 
Table 4 Median LCC values across 1000 train-test trials of the BIQA methods and their enriched version by the proposed aesthetic features on the benchmark databases. Bold and italic cells show the best performance in each row.

\begin{tabular}{|c|c|c|c|c|c|c|c|c|c|c|c|}
\hline \multirow[b]{2}{*}{ Database } & \multirow[b]{2}{*}{ Distortion } & \multicolumn{5}{|c|}{ Original methods } & \multicolumn{5}{|c|}{ Enriched methods with the proposed aesthetic features } \\
\hline & & BRISQUE & SSEQ & M3 & BLIINDS-II & DIIVINE & BRISQUE & SSEQ & M3 & BLIINDS-II & DIIVINE \\
\hline \multirow[t]{6}{*}{ LIVE } & JPEG2000 & 0.9426 & 0.8325 & 0.9501 & 0.9518 & 0.907 & 0.9583 & 0.8918 & 0.9647 & 0.9641 & 0.9254 \\
\hline & JPEG & 0.9829 & 0.9303 & 0.9831 & 0.9706 & 0.9457 & 0.9824 & 0.9609 & 0.9835 & 0.9824 & 0.9646 \\
\hline & WN & 0.9888 & 0.9387 & 0.9927 & 0.9715 & 0.9834 & 0.9851 & 0.9576 & 0.99 & 0.9864 & 0.985 \\
\hline & Gblur & 0.965 & 0.8816 & 0.9504 & 0.9356 & 0.9165 & 0.967 & 0.9315 & 0.9559 & 0.9597 & 0.9289 \\
\hline & Fast fading & 0.9164 & 0.785 & 0.9411 & 0.8941 & 0.8722 & 0.922 & 0.854 & 0.9458 & 0.9271 & 0.883 \\
\hline & Total & 0.9587 & 0.8628 & 0.9657 & 0.9439 & 0.9184 & 0.9627 & 0.9067 & 0.9698 & 0.964 & 0.9355 \\
\hline \multirow[t]{7}{*}{ CSIQ } & Noise & 0.8849 & 0.8266 & 0.7251 & 0.7627 & 0.8867 & 0.9081 & 0.8936 & 0.943 & 0.9057 & 0.9178 \\
\hline & JPEG & 0.9611 & 0.8858 & 0.9604 & 0.9277 & 0.9429 & 0.9667 & 0.9373 & 0.9705 & 0.9614 & 0.9635 \\
\hline & JPEG2000 & 0.9326 & 0.8576 & 0.9537 & 0.9088 & 0.937 & 0.9335 & 0.9133 & 0.9733 & 0.9483 & 0.9363 \\
\hline & Fnoise & 0.9003 & 0.7121 & 0.8392 & 0.6835 & 0.8568 & 0.9414 & 0.8595 & 0.9372 & 0.8863 & 0.8983 \\
\hline & Blur & 0.9339 & 0.8975 & 0.9357 & 0.9375 & 0.9233 & 0.9543 & 0.944 & 0.9492 & 0.9693 & 0.9292 \\
\hline & Contrast & 0.7634 & 0.5206 & 0.7871 & 0.5222 & 0.7417 & 0.846 & 0.8766 & 0.9131 & 0.8984 & 0.8342 \\
\hline & Total & 0.9106 & 0.8178 & 0.8925 & 0.8365 & 0.8818 & 0.9339 & 0.9111 & 0.954 & 0.9363 & 0.9169 \\
\hline \multirow[t]{4}{*}{ IVC } & JPEG & 0.9765 & 0.9324 & 0.9775 & 0.8083 & 0.9641 & 0.9665 & 0.9373 & 0.9765 & 0.8587 & 0.9542 \\
\hline & JPEG2000 & 0.9508 & 0.8663 & 0.9718 & 0.8726 & 0.9621 & 0.9508 & 0.9126 & 0.9775 & 0.9572 & 0.9533 \\
\hline & LAR coding & 0.9384 & 0.8281 & 0.9409 & 0.8319 & 0.961 & 0.9408 & 0.8881 & 0.9413 & 0.8268 & 0.9259 \\
\hline & Total & 0.9432 & 0.8839 & 0.9574 & 0.8101 & 0.9407 & 0.9482 & 0.9058 & 0.9639 & 0.8813 & 0.9226 \\
\hline \multirow[t]{3}{*}{ TOYAMA } & JPEG2000 & 0.9522 & 0.8757 & 0.9472 & 0.94 & 0.8273 & 0.9534 & 0.9186 & 0.9466 & 0.957 & 0.9133 \\
\hline & JPEG & 0.9684 & 0.9363 & 0.9623 & 0.9547 & 0.8887 & 0.9635 & 0.946 & 0.9612 & 0.9504 & 0.9439 \\
\hline & Total & 0.9577 & 0.8978 & 0.9522 & 0.9425 & 0.8505 & 0.9556 & 0.9278 & 0.9512 & 0.9504 & 0.926 \\
\hline TID2008 & Total & 0.7963 & 0.75 & 0.8335 & 0.7666 & 0.7534 & 0.8307 & 0.7772 & 0.8566 & 0.8083 & 0.7884 \\
\hline TID2013 & Total & 0.7887 & 0.7302 & 0.8113 & 0.72 & 0.7683 & 0.8441 & 0.7822 & 0.8561 & 0.7873 & 0.8103 \\
\hline MDID & Total & 0.8992 & 0.8978 & 0.8358 & 0.8463 & 0.9059 & 0.9259 & 0.9352 & 0.8940 & 0.8956 & 0.9238 \\
\hline
\end{tabular}

the better results come from enriching the features of the BIQA methods using the proposed aesthetic features.

As can be seen in Table 4, enriching the BIQA methods, generally but not always, improved the accuracy of the methods. For some distortions such as WN in the LIVE database, the performance almost remains the same after enriching the BIQA method. One possible reason for that is the high accuracy of the original methods for this distortion in the LIVE database. Table 5 illustrates the performance of five tested BIQA methods and their enriched versions on seven benchmark IQA databases based on the SROCC metric. All methods show performance improvements when they are enriched by the proposed aesthetic features.

For better visualization of the improvements made by the proposed aesthetic features, we plotted the improvements of the BIQA methods when they are enriched by our proposed features using bar graphs. Figures 10-16 show the improvements (or deterioration) of the median SROCC values after enriching the testing BIQA methods by the proposed aesthetic features. The reported median values are computed 
Table 5 Median SROCC values across 1000 train-test combinations of the BIQA methods and their enriched version by the proposed aesthetic features on the benchmark IQA databases. Bold and italic cells show the best performance in each row.

\begin{tabular}{|c|c|c|c|c|c|c|c|c|c|c|c|}
\hline \multirow[b]{2}{*}{ Database } & \multirow[b]{2}{*}{ Distortion } & \multicolumn{5}{|c|}{ Original methods } & \multicolumn{5}{|c|}{ Enriched methods with the proposed aesthetic features } \\
\hline & & BRISQUE & SSEQ & M3 & BLIINDS-II & DIIVINE & BRISQUE & SSEQ & M3 & BLIINDS-II & DIIVINE \\
\hline \multirow[t]{6}{*}{ LIVE } & JPEG2000 & 0.9314 & 0.8481 & 0.9345 & 0.9406 & 0.903 & 0.9473 & 0.8931 & 0.9508 & 0.9588 & 0.9199 \\
\hline & JPEG & 0.9745 & 0.9172 & 0.9721 & 0.9579 & 0.9302 & 0.9699 & 0.9493 & 0.9727 & 0.9706 & 0.9508 \\
\hline & WN & 0.981 & 0.9148 & 0.9834 & 0.9536 & 0.9633 & 0.9783 & 0.9358 & 0.9796 & 0.9761 & 0.9671 \\
\hline & Gblur & 0.9637 & 0.833 & 0.9432 & 0.9256 & 0.9207 & 0.9657 & 0.9217 & 0.9517 & 0.9551 & 0.9219 \\
\hline & Fast fading & 0.8948 & 0.7354 & 0.9217 & 0.879 & 0.857 & 0.8907 & 0.8209 & 0.9245 & 0.9047 & 0.8708 \\
\hline & Total & 0.9569 & 0.8501 & 0.9627 & 0.9402 & 0.9194 & 0.9604 & 0.8944 & 0.9676 & 0.961 & 0.9349 \\
\hline \multirow[t]{7}{*}{ CSIQ } & Noise & 0.8774 & 0.8306 & 0.7553 & 0.7519 & 0.8748 & 0.911 & 0.8876 & 0.9342 & 0.8976 & 0.912 \\
\hline & JPEG & 0.9221 & 0.8315 & 0.9077 & 0.8681 & 0.9088 & 0.9464 & 0.9226 & 0.9347 & 0.9276 & 0.9388 \\
\hline & JPEG2000 & 0.9091 & 0.778 & 0.9304 & 0.8655 & 0.9156 & 0.9226 & 0.8812 & 0.9596 & 0.9243 & 0.9281 \\
\hline & Fnoise & 0.9151 & 0.7253 & 0.8408 & 0.685 & 0.8712 & 0.9466 & 0.8706 & 0.9351 & 0.8927 & 0.9163 \\
\hline & Blur & 0.8954 & 0.8352 & 0.8984 & 0.909 & 0.9 & 0.933 & 0.9113 & 0.9222 & 0.9535 & 0.9165 \\
\hline & Contrast & 0.74 & 0.4158 & 0.7493 & 0.4657 & 0.7213 & 0.8239 & 0.8516 & 0.8955 & 0.88 & 0.8129 \\
\hline & Total & 0.8868 & 0.7595 & 0.8653 & 0.7887 & 0.8638 & 0.9227 & 0.8947 & 0.946 & 0.9264 & 0.9123 \\
\hline \multirow[t]{4}{*}{ IVC } & JPEG & 0.9604 & 0.9079 & 0.9631 & 0.7799 & 0.9508 & 0.9504 & 0.925 & 0.9615 & 0.8335 & 0.9442 \\
\hline & JPEG2000 & 0.9132 & 0.8533 & 0.95 & 0.848 & 0.944 & 0.9285 & 0.9126 & 0.9568 & 0.9457 & 0.93 \\
\hline & LAR coding & 0.8982 & 0.7972 & 0.9049 & 0.7912 & 0.9333 & 0.9 & 0.8839 & 0.9023 & 0.8 & 0.9 \\
\hline & Total & 0.925 & 0.8821 & 0.9474 & 0.81 & 0.9373 & 0.9382 & 0.9107 & 0.9516 & 0.8779 & 0.9245 \\
\hline \multirow[t]{3}{*}{ TOYAMA } & JPEG2000 & 0.9355 & 0.8783 & 0.9287 & 0.9359 & 0.8321 & 0.9394 & 0.9188 & 0.9318 & 0.9474 & 0.926 \\
\hline & JPEG & 0.9538 & 0.9148 & 0.938 & 0.9311 & 0.8721 & 0.9472 & 0.9275 & 0.9318 & 0.9262 & 0.9331 \\
\hline & Total & 0.9491 & 0.8971 & 0.9369 & 0.9368 & 0.851 & 0.9467 & 0.929 & 0.9361 & 0.9409 & 0.933 \\
\hline TID2008 & Total & 0.7674 & 0.6944 & 0.8185 & 0.7219 & 0.7169 & 0.8151 & 0.734 & 0.846 & 0.7849 & 0.7676 \\
\hline TID2013 & Total & 0.7496 & 0.6393 & 0.7783 & 0.6343 & 0.7145 & 0.8331 & 0.735 & 0.8386 & 0.7347 & 0.7845 \\
\hline MDID & Total & 0.8921 & 0.8924 & 0.8240 & 0.8371 & 0.8969 & 0.9212 & 0.9304 & 0.8856 & 0.8898 & 0.9181 \\
\hline
\end{tabular}

through 1000 train-test trials in each distortion and over all distortions tested on seven benchmark IQA databases. In these figures, if a bar is greater than zero, it means that enriching the BIQA method improves its performance. It is also observable that enriching the features by the proposed aesthetic features does not always improve the performance of the methods.

Figure 10 shows improvements or degradations of the enriched BIQA methods in comparison with their original versions on the LIVE database. This figure shows that for the BRISQUE method, there are very small improvements (or even deterioration) when we enriched its feature with the aesthetic features where some of the other methods show better performance improvements. This observation may be due to the fact that BRISQUE uses MSCN-based features in the spatial domain to form quality aware features and these features are very discriminative for linear distortions introduced in the LIVE database. Also, the subjective quality scores reported for LIVE are in the form of DMOS which reduces the images' content effect in perceptual quality scores. Therefore, enriching the methods features could not make significant performance improvements. 


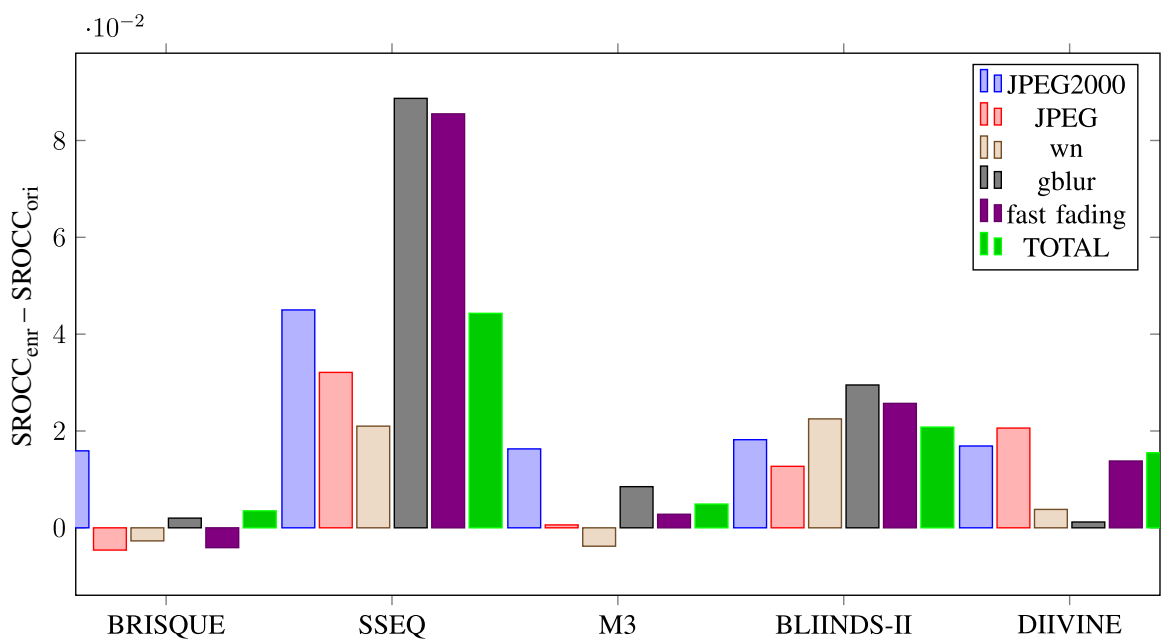

Fig. 10 Performance improvements of median SROCC of the enriched methods across 1000 train-test combinations on the LIVE database. Negative values show degradation in the performances.

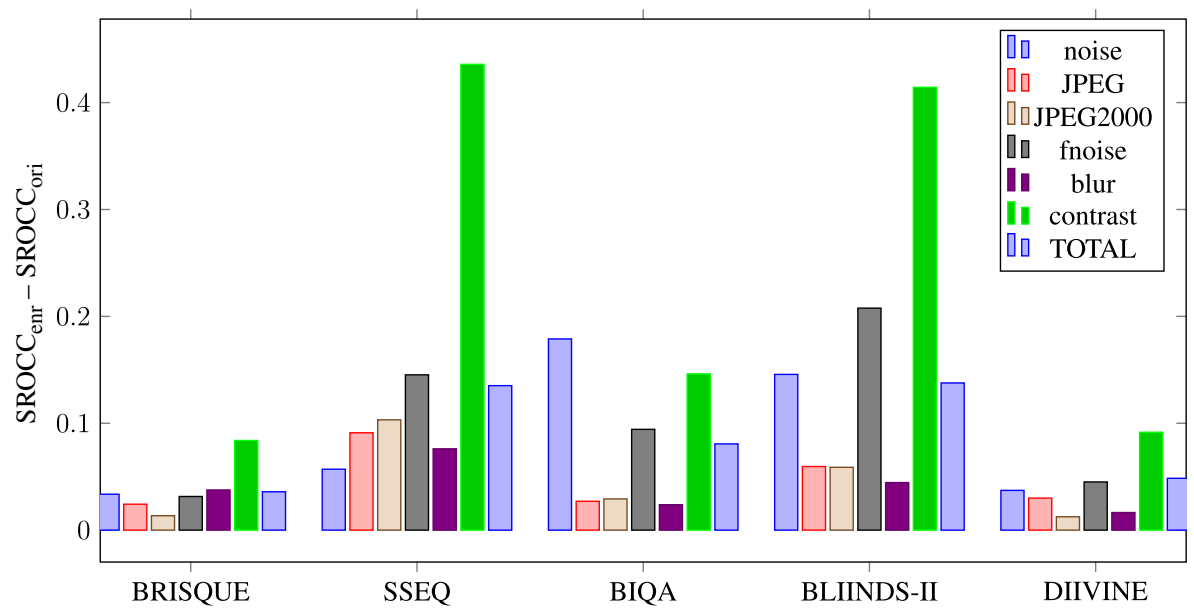

Fig. 11 Performance improvements of median SROCC of the enriched methods across 1000 train-test combinations on the CSIQ database. Negative values show degradation in the performances.

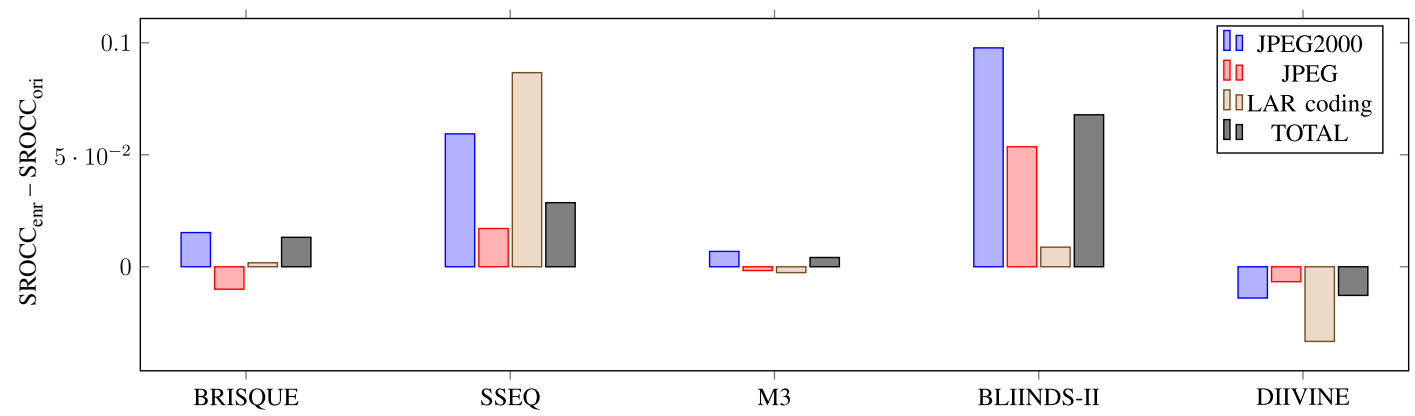

Fig. 12 Performance improvements of median SROCC of the enriched methods across 1000 train-test combinations on the IVC database. Negative values show degradation in the performances.

It is important to note that the ground truth scores in some publicly available databases like the LIVE database are DMOS scores, which are the differences between the MOS scores of the distorted and reference images. In this sense, content dependencies including those related to aesthetics are already reduced. Therefore, for such databases, using aesthetic features may not make significant improvements in the methods performance. But in the tested databases, we observed a significant improvement in CSIQ where its ground truth scores are DMOS scores.

It seems that the content dependencies in CSIQ are not reduced significantly in the DMOS scores. One reason for 


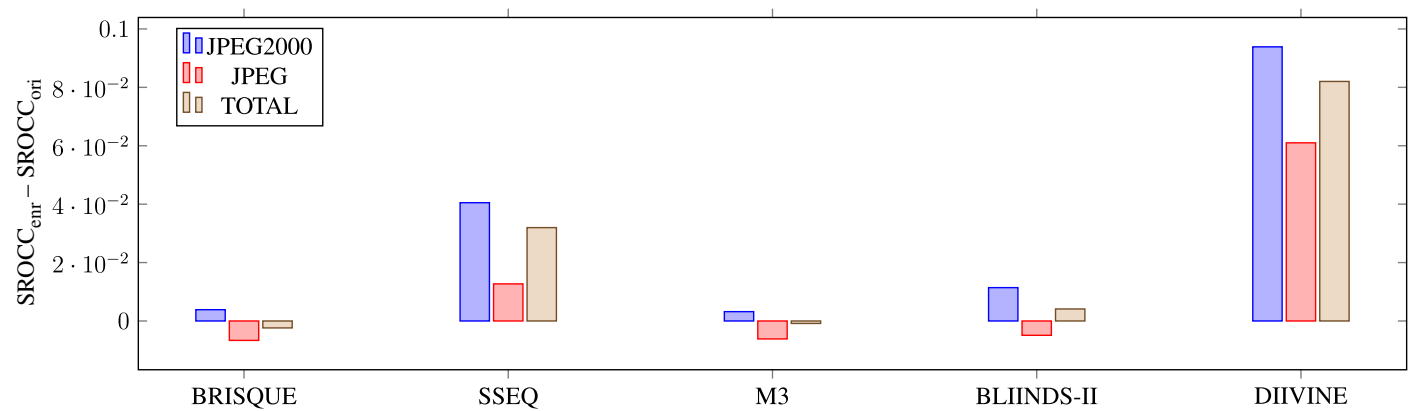

Fig. 13 Performance improvements of median SROCC of the enriched methods across 1000 train-test combinations on the Toyama database. Negative values show degradation in the performances.

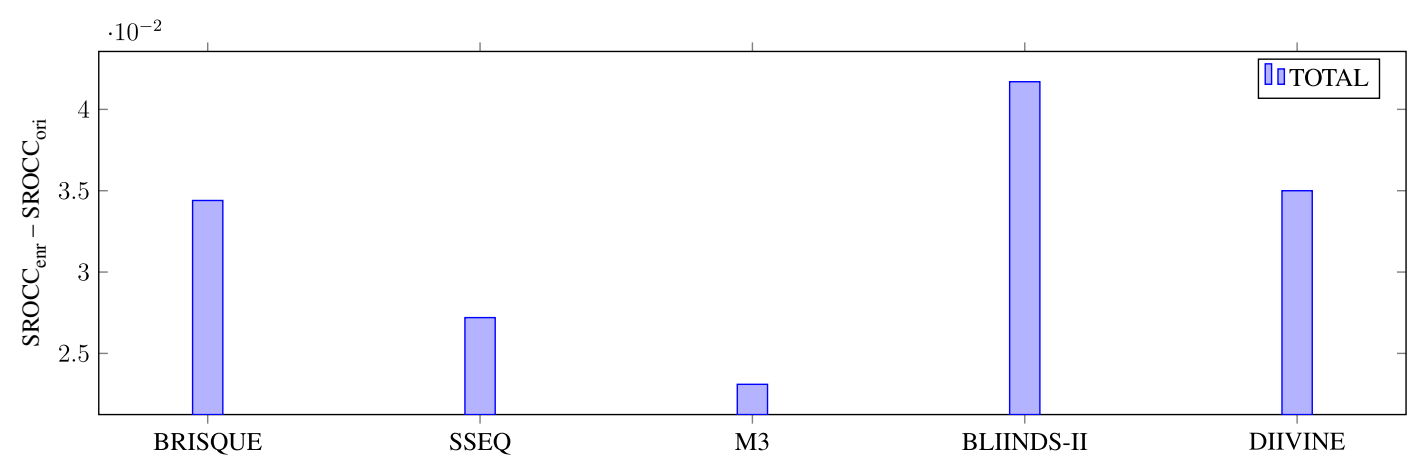

Fig. 14 Performance improvements of median SROCC of the enriched methods across 1000 train-test combinations on the TID2008 database.

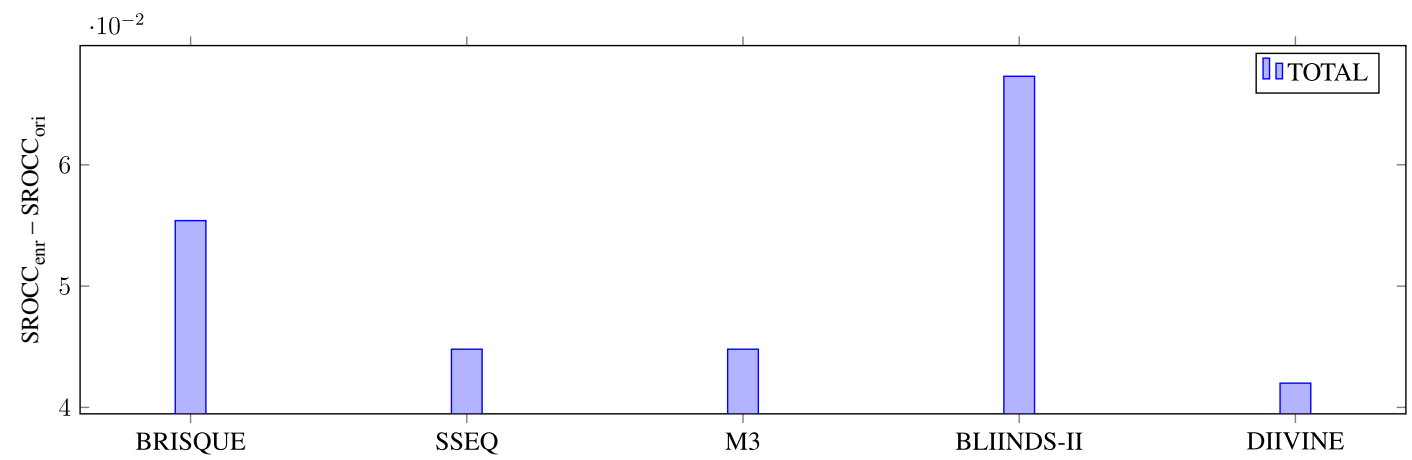

Fig. 15 Performance improvements of median SROCC of the enriched methods across 1000 train-test combinations on the TID2013 database.

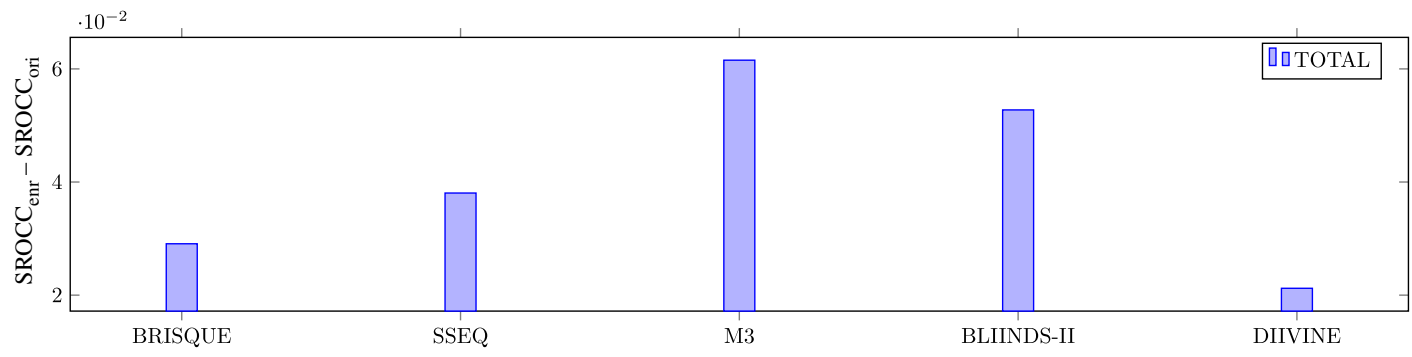

Fig. 16 Performance improvements of median SROCC of the enriched methods across 1000 train-test combinations on the MDID database. 


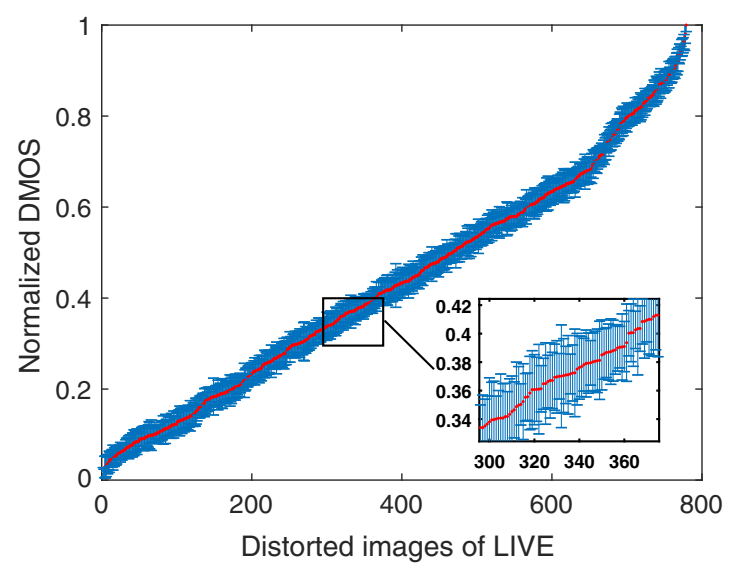

(a)

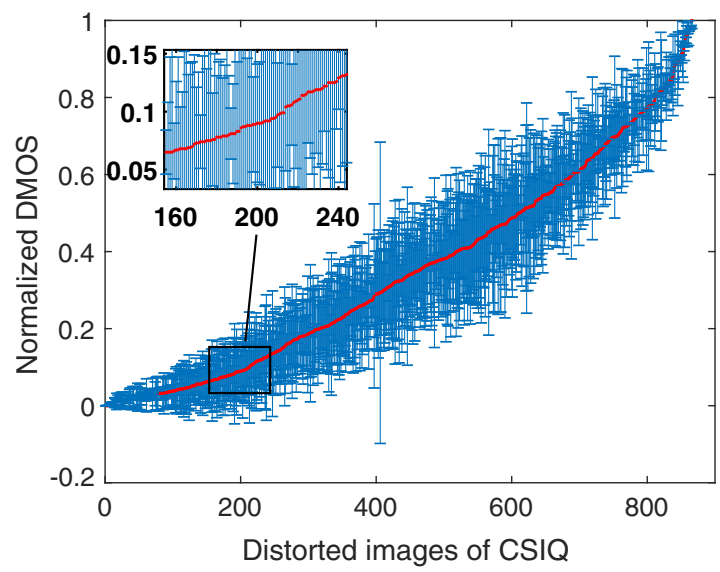

(b)

Fig. 17 Comparison of the normalized standard deviations of DMOSs of (a) LIVE and (b) CSIQ. The DMOSs and the standard deviations are normalized for better visualization.

this observation is that these DMOS scores are not provided with sufficient subjective evaluation. Figure 17 compares the normalized standard deviations of the images for LIVE and CSIQ databases. As can be seen in this figure, the normalized standard deviations of CSIQ are much higher than those in LIVE. Looking to psychometric evaluation conditions of CSIQ in Table 3 shows that in CSIQ only 5 to 7 subjects rated the images and maybe this small number of subjects leads to higher standard deviations, and therefore, computing such DMOS scores did not reduce the content dependency significantly. So, enriching the BIQA methods by the proposed aesthetic features made more performance improvements in CSIQ than LIVE.

Multiple distorted databases like MDID and TID2013 are more challenging for BIQA methods. As can be seen in Figs. 15 and 16, our proposed aesthetic features help the BIQA methods to achieve better performance in the multiple distorted image quality databases. The reported subjective scores in these two databases are in the form of MOS which uses the mean value of the subjects' judgments for each image. Therefore, their scores may be affected by image content. Figures 15 and 16 show that image aesthetics are correlated with human image quality judgment in our experiments. So, enriching the NSS-based features by the proposed aesthetic feature made significant improvements in the BIQA methods performance.

Apparently, all the combinations of methods-distortions did not improve by enriching the BIQA methods using the proposed aesthetic features. The most important thing is that the improvement in the total databases is achieved by enriching the methods, but in some distortion types in some tested databases a better image quality prediction accuracy is not the outcome of enriching the methods. For example, according to Tables 4 and 5, in the case of "white noise" and "JPEG" distortions for the LIVE database, best SROCC values are the outcome of the original version of BRISQUE and M3 methods. But our proposed features help these methods on assessing the quality of images on the same distortions in other databases and also in other distortions for all tested IQA databases.

The reason could be the low diversity in distortions of the LIVE database, which may lead the methods to estimate the quality of some specific distortions very well, meanwhile, making their behavior inexpedient in a real application that usually faces many distortions. BRISQUE method features are formed based on the NSS in the spatial domain called MSCN. The WN image features are mostly separated and have little overlap with other distortion types in the features space. So, the original BRISQUE method has a high performance to estimate the quality of the WN distorted images on the LIVE database and enriching it with the proposed aesthetic features cannot improve its performance on such images.

The proposed features always improved the performance of the state-of-the-art-methods in the CSIQ database for all distortions introduced in this database as can be seen in Fig. 11. Our experiments on IVC and Toyama databases show that the SSEQ and BLIINDS-II methods performances always improved when they are enriched, but for BRISQUE, M3, and DIIVINE methods; their performance degraded in some distortions when they are enriched as can be seen in Figs. 12 and 13 and Table 5.

However, in both Tables 4 and 5, the best correlations on the whole database for all databases except MDID comes from the enriched M3 method. For the MDID database, enriched SSEQ shows a higher correlation value. In the TID2008 database, we conducted experiments on over all distortions of the databases. The results of enriching the tested BIQA methods are illustrated in Fig. 14 and Table 5. The performance of the enriched methods always improved over all 17 distortions introduced in the TID2008 database.

TID2013 and MDID databases are more challenging databases for image quality assessment methods. Each image in these databases is distorted by multiple distortions. As can be seen in Figs. 15 and 16, the proposed aesthetic features improved the performance of all the tested BIQA methods over all distortions introduced in these databases. The enriched BLIINDS-II method by aesthetics features shows high improvements for different databases where the enriched SSEQ method achieved better improvements in challenging databases such as MDID. Our experiments show that the proposed approach is useful for improving the performance of the state-of-the-art methods to predict the subjective quality scores. 


\subsubsection{Training set ratio dependency}

There is a critic on machine learning-based methods and it depends on the training set ratio. We used Eq. (23) with $\alpha=$ 0.5 and regenerated the results by the same approach. Table 6 demonstrates the predicted quality score correlations with the ground truth subjective scores. The highest value in each column for each database is bold in order to imply in most of cases the best results come from augmenting the tested BIQA methods features by the proposed aesthetic features. Overall, changing $\alpha$ effects the predication accuracy of the methods but the performance improvement is significant when we enriched the methods by the proposed aesthetic features as can be seen in Table 6 .

\subsubsection{Statistical significance testing}

To determine whether the performance improvements of the enriched BIQA methods over their original versions are statistically significant, two tests were performed on the SROCC values obtained from the 1000 train-test trials on the challenging MDID database.

The two-sample $t$-test and the Wilcoxon rank-sum test ${ }^{76}$ are conducted to test the mean and median values of two populations, respectively. The two-sample $t$-test examines the equivalence of the mean values of two samples drawn from independent populations of a normal distribution. So, for the $t$-test, independency and normality of the samples data must be guaranteed. The random splitting of the image quality database into training and testing sets for computing each SROCC value ensured the independency of the SROCC values. The empirical distribution of the SROCC values shows that it follows a right-skewed unimodal distribution. To ensure the normality assumption, we transformed the SROCC values by exponentiation, ${ }^{76}$ and then the $t$-test was performed on these transformed data.

The null hypothesis of the $t$-test is that the SROCC values of the (row) method and the SROCC values of the (column) method are drawn from populations with equal mean. The alternative hypothesis is that the mean of one method is greater or lesser than the other.

The Wilcoxon rank-sum test examines the equivalence of the median values of two independent samples and is a nonparametric test. The null hypothesis for the Wilcoxon ranksum test is that the SROCC values of the (row) method and the SROCC values of the (column) method are drawn from populations with equal median value. The alternative hypothesis is that the median value of one method is greater or lesser than the other.

Both tests were performed at a significance level of 0.01 (a confidence of 99\%) using the 1000 SROCC values of all pairs of the enriched and the original versions of the tested BIQA methods.

The results of the two tests are illustrated in Tables 7 and 8 , respectively. A value of " 1 " indicates that the row method is statistically superior in performance over the column method, a value of " -1 " indicates that the row method is statistically inferior in performance than the column method, whereas a value of " 0 " indicates that the method in the row is statistically equivalent in performance to the method in the column with confidence of $99 \%$.

The two tests lead to the same conclusions on the MDID database. Each method was compared with all other enriched and original versions of the BIQA methods for testing the statistical significant improvement of the performance. The results show each enriched BIQA method is statistically superior to its original version. So, the aesthetic features can make significant improvements in the performance of the tested BIQA methods.

As can be seen in Tables 7 and 8, the enriched versions of SSEQ and BRISQUE methods are significantly superior to all other tested enriched and original BIQA methods. The tests also show that the original versions of BRISQUE and SSEQ methods are statistically indistinguishable at a significance level of 0.01 , but by enriching them using

Table 6 Median SROCC values across 1000 train-test trials for some BIQA methods on the benchmark databases. 50\% of image samples are used for training and $50 \%$ of image samples for test. Bold cells show the highest performance for each database.

\begin{tabular}{lcccccccc}
\hline Database & & & & & & & \\
Original methods & BRISQUE & 0.9504 & 0.8619 & 0.732 & 0.7376 & 0.8855 & $\mathbf{0 . 9 2 7}$ & 0.8575 \\
& SSEQ & 0.8441 & 0.748 & 0.6287 & 0.6811 & 0.8526 & 0.8724 & 0.8529 \\
& M3 & 0.959 & 0.8491 & 0.7645 & 0.7956 & 0.9268 & 0.9145 & 0.8048 \\
& BLIINDS-II & 0.9345 & 0.7766 & 0.6241 & 0.7018 & 0.715 & 0.9183 & 0.8025 \\
& DIIVINE & 0.9067 & 0.843 & 0.698 & 0.6901 & 0.8868 & 0.8457 & 0.8479 \\
\hline Enriched methods & BRISQUE & 0.9494 & 0.9018 & 0.8083 & 0.7662 & 0.8953 & 0.9194 & 0.8945 \\
& SSEQ & 0.8824 & 0.8791 & 0.7143 & 0.7054 & 0.8577 & 0.8873 & $\mathbf{0 . 9 0 7 6}$ \\
& MLIINDS-II & 0.9533 & 0.9101 & 0.7124 & 0.7475 & 0.8129 & 0.9193 & 0.8592 \\
& DIIVINE & 0.924 & 0.8926 & 0.7689 & 0.7448 & 0.8902 & 0.9192 & 0.8635 \\
\hline
\end{tabular}


Table 7 Results of the two-sample $t$-test performed by using the SROCC values of the enriched BIQA methods and their original versions. A value of 1 indicates that the row method is statistically superior to the column method, a value of 1 indicates that the row method is statistically inferior to the column method, whereas a value of 0 indicates that they are statistically indistinguishable in performance. The test performed at a significance level of 0.01 .

\begin{tabular}{|c|c|c|c|c|c|c|c|c|c|c|c|}
\hline \multirow[b]{2}{*}{ MDID database } & & \multicolumn{5}{|c|}{ Original BIQA methods } & \multicolumn{5}{|c|}{ Enriched BIQA methods } \\
\hline & & BRISQUE & SSEQ & M3 & BLIINDS-II & DIIVINE & BRISQUE & SSEQ & M3 & BLIINDS-II & DIIVINE \\
\hline \multirow[t]{5}{*}{ Original BIQA methods } & BRISQUE & 0 & 0 & 1 & 1 & -1 & -1 & -1 & 1 & 1 & -1 \\
\hline & SSEQ & 0 & 0 & 1 & 1 & -1 & -1 & -1 & 1 & 1 & -1 \\
\hline & M3 & -1 & -1 & 0 & -1 & -1 & -1 & -1 & -1 & -1 & -1 \\
\hline & BLIINDS-II & -1 & -1 & 1 & 0 & -1 & -1 & -1 & -1 & -1 & -1 \\
\hline & DIIVINE & 1 & 1 & 1 & 1 & 0 & -1 & -1 & 1 & 1 & -1 \\
\hline \multirow[t]{5}{*}{ Enriched BIQA Methods } & BRISQUE & 1 & 1 & 1 & 1 & 1 & 0 & -1 & 1 & 1 & 1 \\
\hline & SSEQ & 1 & 1 & 1 & 1 & 1 & 1 & 0 & 1 & 1 & 1 \\
\hline & M3 & -1 & -1 & 1 & 1 & -1 & -1 & -1 & 0 & -1 & -1 \\
\hline & BLIINDS-II & -1 & -1 & 1 & 1 & -1 & -1 & -1 & 1 & 0 & -1 \\
\hline & DIIVINE & 1 & 1 & 1 & 1 & 1 & -1 & -1 & 1 & 1 & 0 \\
\hline
\end{tabular}

Table 8 Results of the Wilcoxon rank-sum test performed by using the SROCC values of the enriched BIQA methods and their original versions. A value of 1 indicates that the row method is statistically superior to the column method, a value of 1 indicates that the row method is statistically inferior to the column method, whereas a value of 0 indicates that they are statistically indistinguishable in performance. The test performed at a significance level of 0.01 .

\begin{tabular}{|c|c|c|c|c|c|c|c|c|c|c|c|}
\hline \multirow[b]{2}{*}{ MDID database } & & \multicolumn{5}{|c|}{ Original BIQA methods } & \multicolumn{5}{|c|}{ Enriched BIQA methods } \\
\hline & & BRISQUE & SSEQ & M3 & BLIINDS-II & DIIVINE & BRISQUE & SSEQ & M3 & BLIINDS-II & DIIVINE \\
\hline \multirow[t]{5}{*}{ Original BIQA methods } & BRISQUE & 0 & 0 & 1 & 1 & -1 & -1 & -1 & 1 & 1 & -1 \\
\hline & SSEQ & 0 & 0 & 1 & 1 & -1 & -1 & -1 & 1 & 1 & -1 \\
\hline & M3 & -1 & -1 & 0 & -1 & -1 & -1 & -1 & -1 & -1 & -1 \\
\hline & BLIINDS-II & -1 & -1 & 1 & 0 & -1 & -1 & -1 & -1 & -1 & -1 \\
\hline & DIIVINE & 1 & 1 & 1 & 1 & 0 & -1 & -1 & 1 & 1 & -1 \\
\hline \multirow[t]{5}{*}{ Enriched BIQA methods } & BRISQUE & 1 & 1 & 1 & 1 & 1 & 0 & -1 & 1 & 1 & 1 \\
\hline & SSEQ & 1 & 1 & 1 & 1 & 1 & 1 & 0 & 1 & 1 & 1 \\
\hline & M3 & -1 & -1 & 1 & 1 & -1 & -1 & -1 & 0 & -1 & -1 \\
\hline & BLIINDS-II & -1 & -1 & 1 & 1 & -1 & -1 & -1 & 1 & 0 & -1 \\
\hline & DIIVINE & 1 & 1 & 1 & 1 & 1 & -1 & -1 & 1 & 1 & 0 \\
\hline
\end{tabular}

the proposed aesthetic features, the enriched SSEQ method is superior to the enriched BRISQUE method.

Figure 16 shows the improvement of the tested BIQA methods after enriching them by the proposed aesthetic features tested on the MDID database.

\subsubsection{Database dependency analysis}

To analyze the train database dependency on the performance of enriching BIQA methods, we trained the methods in one benchmark image quality database and tested them in another database. Table 9 shows the performance 
Table 9 SROCC values when TID2013 used as training set and LIVE for test set. Bold cells have the highest score in the whole row and the best score of each method for each distortion has been made italic.

\begin{tabular}{|c|c|c|c|c|c|c|c|c|c|c|c|}
\hline \multirow[b]{2}{*}{ Database } & \multirow[b]{2}{*}{ Distortion } & \multicolumn{5}{|c|}{ Original methods } & \multicolumn{5}{|c|}{ Enriched methods with the proposed aesthetic features } \\
\hline & & BRISQUE & SSEQ & M3 & BLIINDS-II & DIIVINE & BRISQUE & SSEQ & M3 & BLIINDS-II & DIIVINE \\
\hline \multirow[t]{6}{*}{ LIVE } & JPEG2000 & 0.694 & 0.5142 & 0.7368 & 0.6915 & 0.7206 & 0.7389 & 0.7483 & 0.7849 & 0.758 & 0.7935 \\
\hline & JPEG & 0.8664 & 0.8109 & 0.8975 & 0.8725 & 0.891 & 0.896 & 0.8309 & 0.8844 & 0.8529 & 0.8786 \\
\hline & WN & 0.9482 & 0.6082 & 0.9423 & 0.9401 & 0.8874 & 0.9517 & 0.6988 & 0.9643 & 0.9472 & 0.9288 \\
\hline & Gblur & 0.5446 & 0.5578 & 0.6733 & 0.7807 & 0.7797 & 0.8747 & 0.7446 & 0.8537 & 0.9202 & 0.8536 \\
\hline & Fast fading & 0.7164 & 0.6016 & 0.8236 & 0.6968 & 0.8351 & 0.7673 & 0.6581 & 0.8397 & 0.7482 & 0.8271 \\
\hline & Total & 0.7758 & 0.664 & 0.836 & 0.7994 & 0.8297 & 0.8303 & 0.755 & 0.8671 & 0.8342 & 0.8339 \\
\hline
\end{tabular}

of the original version of five BIQA methods and their enriched versions when we used all images of TID2013 with the same distortion types with LIVE as a training set and tested the methods by the LIVE database. The left part of the table shows the performance of the original methods in each distortion and also over all distortions and the right part of the table shows the performance of the enriched methods by the proposed aesthetic features. Table 9 illustrates that some of the methods like BLIIND-II and M3 show a higher performance when they were trained by TID2013 and tested by LIVE. When the methods enriched by the proposed aesthetic features, their performance almost improved in all distortions in all methods except for JPEG distortions.

\section{Conclusion}

In this paper, we proposed a host of aesthetic features to augment the existing NSS-based BIQA methods. Also, we modified an application of Benford's law, Machado and Cardoso, and PC such that better correlation with subjective quality was achieved. We enriched the features of five stateof-the-art BIQA methods by augmenting their features of natural image statistics with our proposed aesthetic features. Two different experiments have been carried out to support the argument that the observed performance improvements in the enriched BIQA methods are due to the aesthetic features based on feature section and PCA approaches. Then, extensive experiments showed significant improvements on the performance of the enriched NSS-based BIQA methods. We have conducted a two-sample $t$-test and Wilcoxon rank-sum tests to show that the observed improvements, which were achieved by augmenting the BIQA features are significant.

In this work, the proposed features were used to enrich the opinion-aware NSS-based BIQA methods. In our future works, the aesthetic features will be used to also improve the performance of opinion-unaware completely blind IQA methods. Also, we will study the effect of image emotion as well as image content in image and video quality assessment databases with authentic distortions. ${ }^{77}$ For this aim, we will use the DNNs to extract content and emotion aware features.

\section{Acknowledgments}

We would like to thank the German Research Foundation (DFG) for financial support within project A05 of SFB/ Transregio 161. Also, we would like to thank the anonymous reviewers for their very helpful comments and Dietmar Saupe for proofreading of this paper.

\section{References}

1. Z. Chen, T. Jiang, and Y. Tian, "Quality assessment for comparing image enhancement algorithms," in IEEE Conf. on Computer Vision and Pattern Recognition (CVPR), pp. 3003-3010 (2014).

2. C. Lu, L. Xu, and J. Jia, "Contrast preserving decolorization with perception-based quality metrics," Int. J. Comput. Vision 110(2), 222-239 (2014).

3. Z. Sun and A. Hoogs, "Image comparison by compound disjoint information with applications to perceptual visual quality assessment, image registration and tracking," Int. J. Comput. Vision 88(3), 461-488 (2010).

4. N. Nill, "A visual model weighted cosine transform for image compression and quality assessment," IEEE Trans. Commun. 33(6), 551-557 (1985).

5. K. H. Talukder and K. Harada, "Haar wavelet based approach for image compression and quality assessment of compressed image," arXiv preprint arXiv:1010.4084 (2010).

6. W. Griffin and M. Olano, "Objective image quality assessment of texture compression," in I3D Proc. of the 18th Meeting of the ACM SIGGRAPH Symp. on Interactive 3D Graphics and Games, pp. 119126, ACM, New York (2014).

7. A. Mittal, A. Moorthy, and A. Bovik, "No-reference image quality assessment in the spatial domain," IEEE Trans. Image Process. 21(12), 4695-4708 (2012).

8. A. Mittal, R. Soundararajan, and A. Bovik, "Making a completely blind image quality analyzer," IEEE Signal Process. Lett. 20(3), 209-212 (2013).

9. A. Moorthy and A. Bovik, "Blind image quality assessment: from natural scene statistics to perceptual quality," IEEE Trans. Image Process. 20(12), 3350-3364 (2011).

10. M. Saad, A. Bovik, and C. Charrier, "Blind image quality assessment: a natural scene statistics approach in the DCT domain," IEEE Trans. Image Process. 21(8), 3339-3352 (2012).

11. W. Xue et al., "Blind image quality assessment using joint statistics of gradient magnitude and laplacian features," IEEE Trans. Image Process. 23(11), 4850-4862 (2014).

12. M. Jenadeleh and M. E. Moghaddam, "BIQWS: efficient Wakeby modeling of natural scene statistics for blind image quality assessment," Multimedia Tools Appl. 76(12), 13859-13880 (2017).

13. The Editors of Encyclopædia Britannica, "Quality," Encyclopædia Britannica, Inc., 2009, http://www.britannica.com/topic/qualityphilosophy (20 August 2015).

14. N. Ponomarenko et al., "Color image database TID2013: peculiarities and preliminary results," in 4th European Workshop on Visual Information Processing (EUVIP), pp. 106-111 (2013).

15. E. Siahaan, A. Hanjalic, and J. A. Redi, "Augmenting blind image quality assessment using image semantics," in IEEE Int. Symp. on Multimedia (ISM), pp. 307-312, IEEE (2016). 
16. A. Torralba, K. P. Murphy, and W. T. Freeman, "Using the forest to see the trees: exploiting context for visual object detection and localization," Commun. ACM 53(3), 107-114 (2010).

17. J. A. Redi, "Visual quality beyond artifact visibility," Proc. SPIE 8651, $86510 \mathrm{~N}(2013)$.

18. A. Saha and Q. M. J. Wu, "Utilizing image scales towards totally training free blind image quality assessment," IEEE Trans. Image Process. 24(6), 1879-1892 (2015).

19. F. Kerouh and A. Serir, "A perceptual blind blur image quality metric," in IEEE Int. Conf. on Acoustics, Speech and Signal Processing (ICASSP), pp. 2784-2788, IEEE (2014).

20. $\mathrm{L}$. Li et al., "No-reference image blur assessment based on discrete orthogonal moments," IEEE Trans. Cybern. 46, 39-50 (2015).

21. T. Oh and S. Lee, "Blind sharpness prediction based on image-based motion blur analysis," IEEE Trans. Broadcast. 61(1), 1-15 (2015).

22. A. B. Lamb and M. Khambete, "Perceived no reference image quality measurement for chromatic aberration," J. Electron. Imaging 25(2), 023004 (2016).

23. S. A. Golestaneh and D. M. Chandler, "No-reference quality assessment of JPEG images via a quality relevance map," IEEE Signal Process. Lett. 21(2), 155-158 (2014).

24. P. Gastaldo et al., "No-reference quality assessment of JPEG images by using CBP neural networks," in Proc. of the 17th Int. Conf. on Artificial Neural Networks, pp. 564-572, Springer (2007).

25. H. R. Sheikh, A. C. Bovik, and L. Cormack, "Blind quality assessment of JPEG2000 compressed images using natural scene statistics," in Thirty-Seventh Asilomar Conf. on Signals, Systems and Computers, Vol. 2, pp. 1403-1407, IEEE (2003).

26. J. Zhang and T. M. Le, "A new no-reference quality metric for JPEG2000 images," IEEE Trans. Consum. Electron. 56(2), 743-750 (2010).

27. J. Shen, Q. Li, and G. Erlebacher, "Hybrid no-reference natural image quality assessment of noisy, blurry, JPEG2000, and JPEG images," IEEE Trans. Image Process. 20(8), 2089-2098 (2011).

28. K. Gu et al., "Hybrid no-reference quality metric for singly and multiply distorted images," IEEE Trans. Broadcast. 60(3), 555-567 (2014).

29. M. Jenadeleh and M. E. Moghaddam, "Blind image quality assessment through Wakeby statistics model," in Int. Conf. Image Analysis and Recognition, pp. 14-21, Springer (2015).

30. A. K. Moorthy and A. C. Bovik, "A two-step framework for constructing blind image quality indices," IEEE Signal Process. Lett. 17(5), 513516 (2010).

31. L. He et al., "Sparse representation for blind image quality assessment," in IEEE Conf. on Computer Vision and Pattern Recognition (CVPR), pp. 1146-1153, IEEE (2012).

32. Y. Chu et al., "Blind image quality assessment using statistical independence in the divisive normalization transform domain," J. Electron. Imaging 24(6), 063008 (2015).

33. L. Liu et al., "No-reference image quality assessment in curvelet domain," Signal Process. Image Commun. 29(4), 494-505 (2014).

34. Y. Li et al., "No-reference image quality assessment using statistical characterization in the shearlet domain," Signal Process. Image Commun. 29(7), 748-759 (2014).

35. P. Ye and D. Doermann, "No-reference image quality assessment using visual codebooks," IEEE Trans. Image Process. 21(7), 3129-3138 (2012).

36. C. $\mathrm{Li}, \mathrm{A}$. C. Bovik, and $\mathrm{X}$. Wu, "Blind image quality assessment using a general regression neural network," IEEE Trans. Neural Networks 22(5), 793-799 (2011).

37. Q. Wu et al., "Blind image quality assessment based on multi-channel features fusion and label transfer," IEEE Trans. Circuits Syst. Video Technol. 26(3), 425-440 (2015).

38. X. Gao et al., "Universal blind image quality assessment metrics via natural scene statistics and multiple kernel learning," IEEE Trans. Neural Networks Learn. Syst. 24(12), 2013-2026 (2013).

39. W. Hou et al., "Blind image quality assessment via deep learning," IEEE Trans. Neural Networks Learn. Syst. 26(6), 1275-1286 (2015).

40. W. Xue, L. Zhang, and X. Mou, "Learning without human scores for blind image quality assessment," in IEEE Conf. on Computer Vision and Pattern Recognition (CVPR), pp. 995-1002, IEEE (2013).

41. L. Zhang, L. Zhang, and A. Bovik, "A feature-enriched completely blind image quality evaluator," IEEE Trans. Image Process. 24(8), 2579-2591 (2015).

42. E. den Heijer and A. Eiben, "Comparing aesthetic measures for evolutionary art," in Int. Conf. Applications of Evolutionary Computation, pp. 311-320, Springer (2010).

43. Y. Li, "Adaptive learning evaluation model for evolutionary art," in IEEE Congress on Evolutionary Computation (CEC), pp. 1-8 (2012).

44. E. den Heijer and A. Eiben, "Investigating aesthetic measures for unsupervised evolutionary art," Swarm Evol. Comput. 16, 52-68 (2014).

45. E. den Heijer, "Evolving symmetric and balanced art," in Computational Intelligence, K. Madani, Ed., pp. 33-47, Springer, Heidelberg (2015)

46. X. Tang, W. Luo, and X. Wang, "Content-based photo quality assessment," IEEE Trans. Multimedia 15(8), 1930-1943 (2013).
47. L. Yao et al., "OSCAR: on-site composition and aesthetics feedback through exemplars for photographers," Int. J. Comput. Vision 96(3), $353-383$ (2012)

48. L. Guo et al., "Image esthetic assessment using both hand-crafting and semantic features," Neurocomputing 143, 14-26 (2014).

49. C. Li and T. Chen, "Aesthetic visual quality assessment of paintings," IEEE J. Sel. Top. Signal Process. 3(2), 236-252 (2009).

50. T. M. Hamdani et al., Multi-Objective Feature Selection with NSGA II, pp. 240-247, Springer, Heidelberg (2007).

51. E. C. Larson and D. M. Chandler, "Most apparent distortion: full-reference image quality assessment and the role of strategy," J. Electron. Imaging 19(1), 011006 (2010).

52. H. Sheikh, M. Sabir, and A. Bovik, "A statistical evaluation of recent full reference image quality assessment algorithms," IEEE Trans. Image Process. 15(11), 3440-3451 (2006).

53. J. Walthoe, "Looking out for number one" 1999, https://plus.maths.org/ content/looking-out-number-one (March 2015)

54. B. Spehar et al., "Universal aesthetic of fractals," Comput. Graphics 27(5), 813-820 (2003).

55. P. Machado and A. Cardoso, "Computing aesthetics," in Advances in Artificial Intelligence, pp. 219-228, Springer (1998)

56. L. Rosa, "Image compression" (2004), http://www.mathworks.com/ matlabcentral/fileexchange/4772-image-compression (November 2015).

57. K. Matkovic et al., "Global contrast factor-a new approach to image contrast," in Computational Aesthetics, L. Neumann et al., Eds., pp. 159-168, Eurographics Association, Girona (2005).

58. B. Ross, W. Ralph, and H. Zong, "Evolutionary image synthesis using a model of aesthetics," in IEEE Int. Conf. on Evolutionary Computation, pp. 1087-1094 (2006).

59. K. Sharifi and A. Leon-Garcia, "Estimation of shape parameter for generalized Gaussian distributions in subband decompositions of video," IEEE Trans. Circuits Syst. Video Technol. 5(1), 52-56 (1995).

60. K. He, J. Sun, and X. Tang, "Single image haze removal using dark channel prior," IEEE Trans. Pattern Anal. Mach. Intell. 33(12), 2341-2353 (2011).

61. C. T. Vu, T. D. Phan, and D. M. Chandler, "S $\mathrm{S}_{3}$ : a spectral and spatial measure of local perceived sharpness in natural images," IEEE Trans. Image Process. 21(3), 934-945 (2012).

62. D. Hasler and S. E. Suesstrunk, "Measuring colorfulness in natural images," Proc. SPIE 5007, 87 (2003).

63. D. Cohen-Or et al., "Color harmonization," ACM Trans. Graph. 25(3), 624-630 (2006).

64. P. Le Callet and F. Autrusseau, "Subjective quality assessment IRCCYN/IVC database" (2005), http://www2.irccyn.ec-nantes.fr/ivcdb/

65. IT Union, Methodology for the Subjective Assessment of the Quality of Television Pictures, International Telecommunication Union, Geneva, Switzerland (2003).

66. Y. Horita et al., "MICT image quality evaluation database" (2011) http:// mict.eng.u-toyama.ac.jp/mictdb.html (September 2013).

67. N. Ponomarenko et al., "TID2008-a database for evaluation of fullreference visual quality assessment metrics," Adv. Mod. Radioelectron. 10(4), 30-45 (2009).

68. W. Sun, F. Zhou, and Q. Liao, "MDID: a multiply distorted image database for image quality assessment," Pattern Recognit. 61,153-168 (2017).

69. P. G. Engeldrum, Psychometric Scaling: A Toolkit for Imaging Systems Development, Imcotek Press, Winchester (2000).

70. H. H. Barrett et al., "Model observers for assessment of image quality," Proc. Natl. Acad. Sci. U. S. A. 90(21), 9758-9765 (1993).

71. J.-B. Martens and M. Boschman, "The psychophysical measurement of image quality," in Vision Models and Applications to Image and Video Processing, pp. 69-97, Springer (2001).

72. Z. Wang and E. P. Simoncelli, "Stimulus synthesis for efficient evaluation and refinement of perceptual image quality metrics," Proc. SPIE 5292, 99 (2004)

73. D. M. Chandler, "Seven challenges in image quality assessment: past, present, and future research," ISRN Signal Process. 2013, 905685 (2013).

74. L. Liu et al., "No-reference image quality assessment based on spatial and spectral entropies," Signal Process. Image Commun. 29(8), 856863 (2014).

75. C.-C. Chang and C.-J. Lin, "LIBSVM: a library for support vector machines," ACM Trans. Intell. Syst. Technol. 2, 27:1-27:27 (2011).

76. D. J. Sheskin, Handbook of Parametric and Nonparametric Statistical Procedures, CRC Press, Boca Raton (2003).

77. V. Hosu et al., "The Konstanz natural video database (KoNViD-1k)," in Ninth Int. Conf. on Quality of Multimedia Experience (QoMEX), pp. 1-6 (2017).

Mohsen Jenadeleh received his MSc degree in computer engineering from the Faculty of Computer Science and Engineering, Shahid Beheshti University, G. C., Tehran, Iran, under supervision of Prof. Mohsen Ebrahimi Moghaddam and is currently pursuing his PhD. 
$\mathrm{He}$ is currently working at Multimedia Signal Processing Group, University of Konstanz, Germany, under supervision of Prof. Dietmar Saupe. His research interests include image and video quality assessment, iris recognition systems, image forensics, and color image processing.

Mohammad Masood Masaeli received his BSc degree in computer engineering from Sheikh Bahaei University, Isfahan, Iran, his MSc degree from Shahid Beheshti University, G. C., Tehran, Iran, under supervision of Dr. Mohsen Ebrahimi Moghaddam. He is currently a director of the Software Department, Toorintan Co., Tehran, Iran.
His research interests include image quality assessment, computational photography, and computer vision.

Mohsen Ebrahimi Moghaddam received his BSc, MSc, and PhD degrees from Sharif University, Iran. Since 2006, he has been an associate professor at the Faculty of Computer Science and Engineering, Shahhid Beheshti University, Iran. His research interests are image processing and pattern recognition especially using artificial intelligence techniques such as image retrieval, image security, image quality assessment, and gait recognition. There are several publications in this scope by him and his students. 